\title{
DIFFERENTIAL INEQUALITIES OF CONTINUOUS FUNCTIONS AND REMOVING SINGULARITIES OF RADO TYPE FOR $J$-HOLOMORPHIC MAPS
}

\author{
XIANGHONG GONG and JEAN-PIERRE ROSAY*
}

\section{Introduction}

Among the results of Part One we have:

Proposition A. Let $\Omega$ be an open set in $\mathrm{C}^{n}$ and $f: \Omega \rightarrow C$ be a continuous map. If on $\Omega \backslash f^{-1}(0)|\bar{\partial} f| \leq K|f|$ ( $K$ a positive constant $)$, then $f^{-1}(0)$ is an analytic set.

Among the results of Part Two there is the following:

Proposition B. Let $J$ be a $C^{1}$-smooth almost complex structure defined in $\mathrm{R}^{2 n}$. Let $v$ be a continuous map from the unit disc $\mathrm{D} \subset \mathrm{C}$ into $\left(\mathrm{R}^{2 n}, J\right)$. Let $u$ be either a constant map from $\mathrm{D}$ into $\mathrm{R}^{2 n}$ or a proper J-holomorphic and $\mathscr{C}^{2}$-smooth map from $\mathrm{D}$ into an open subset $\Omega$ of $\mathrm{R}^{2 n}$. Assume that $v$ is $J$-holomorphic near $z$ if $v(z) \notin u(\mathrm{D})$. If $u \equiv u(0)$ then $v$ is J-holomorphic on $\mathrm{D}$; if $u \neq \equiv u(0)$ and $v(0) \in u(\mathrm{D})$, either $v$ maps a neighborhood of 0 into $u(\mathrm{D})$, or $v$ is $J$-holomorphic near 0 .

If $u \equiv u(0)$ the hypothesis is thereof that $v$ is $J$-holomorphic at any point $z$ such that $v(z) \neq u(0)$. Proposition B generalizes the classical Rado Theorem.

The more general statements in the text that contain Propositions A and B are Theorem $\mathrm{A}$ and Theorem $\mathrm{C}$.

Parts One and Two are independent. A unifying theme is the elimination of exceptional sets on which no a-priori assumption is to be made, in the style of Rado's Theorem. In some of our statements this is the only novelty. Another common feature is the role played by differential inequality of the type $|\bar{\partial} f| \leq|f|$ or $|\bar{\partial} f| \leq \epsilon|\partial f|$.

\footnotetext{
* Research of both authors are supported in part by NSF grants.

Received November 8, 2005; in revised form May 14, 2006.
} 


\section{Organization of the paper}

Part One

1. $|\bar{\partial} f| \leq|f|$ versus $\bar{\partial} f=A f$. Analyticity of zero set of $f$ and a counterexample

2. One dimensional vector-valued case

3. Proof of the analyticity of $f^{-1}(0)$ (scalar-valued $f$ defined on $\mathrm{C}^{n}$ )

4. Remarks on uniqueness results on $J$-holomorphic curves

Part Two

1. Removability of polar sets for $J$-holomorphic curves

2. Rado type theorem for $J$-holomorphic curves

\section{Part 1. Analyticity of zero sets of continuous function}

\section{1. $\bar{\partial}$-differential inequalities and results of Part One}

Let $f$ be a continuous function on an open subset $\Omega$ of C. Denote $\Omega^{*}=$ $\Omega \backslash f^{-1}(0)$. By the $\bar{\partial}$-differential inequality $|\bar{\partial} f| \leq K|f|$ on $\Omega^{*}$, we mean that on $\Omega^{*}, \bar{\partial} f=A f$ holds in the distribution sense, and $A \in L_{(0,1)}^{\infty}\left(\Omega^{*}\right)$.

In general, one may, of course, consider a continuous map $f: \Omega \rightarrow \mathrm{C}^{m}$ satisfying $\bar{\partial} f=A f$ on $\Omega^{*}=\Omega \backslash f^{-1}(0)$, where $\Omega$ is now an open subset of $C^{n}$ and $A$ is an $m \times m$ matrix of $(0,1)$-forms on $\Omega^{*}$. We are interested in the analyticity of the zero set $f^{-1}(0)$.

We formulate our results in following three cases: (i) $f$ is a vector-valued continuous function on C, (ii) $f$ is a continuous function on $\mathbf{C}^{n}$, (iii) $f$ is a vector-valued continuous function on $\mathrm{C}^{n}$ under the additional assumption that $f^{-1}(0)$ is already real analytic.

The result for case (iii) will be given later by Proposition 3.4. Here we describe results for (i) and (ii).

(i) Vector-valued $f$, defined on $\mathrm{C}$.

Our first result is the following:

Proposition C. Let $\Omega$ be a connected open subset of $\mathrm{C}$, and let $f: \Omega \rightarrow \mathrm{C}^{m}$ be a continuous map. Assume that $\bar{\partial} f=A f$ on $\Omega \backslash f^{-1}(0)$, where $A$ is an $m \times m$ matrix with entries in $L_{(0,1)}^{p}\left(\Omega \backslash f^{-1}(0)\right)$. If $2<p \leq \infty$ and $f \not \equiv 0$, then $f$ has isolated zeros in $\Omega$ and $\bar{\partial} f=A f$ holds on $\Omega$, where $A$ is set to be zero on $f^{-1}(0)$.

(ii) Scalar-valued $f$, defined on $\mathrm{C}^{n}$. 
Next we turn to continuous functions $f$ on an open subset $\Omega$ of $C^{n}$ with $n>1$. Our approach is based on the above one-dimensional result, by applying it to all complex lines $L$ parallel to a coordinate axis in $C^{n}$. Thus one needs to understand the tedious question about the restriction of $\bar{\partial} f=A f$ to all $z_{j}$-lines, including the restriction of $|\bar{\partial} f| \leq K|f|$ to lines, needed for Proposition A. (Of course, $\bar{\partial}(f \mid L)$ makes sense since $f$ is continuous.)

We postpone this technicality to a later discussion. Here we ask that for every complex line $L$ (intersecting $\Omega$ and) parallel to a coordinate axis, in the distribution sense

$$
\bar{\partial}\left(\left.f\right|_{L}\right)=A_{L} f \text { on } L \cap \Omega \backslash f^{-1}(0), \quad\left\|A_{L}\right\|_{L^{p}\left(L \cap \Omega \backslash f^{-1}(0)\right)} \leq M,
$$

where $M>0$ is a constant (independent of $L$ ).

Equivalently, after we prove Proposition $\mathrm{C}$ in section 2, the above equation can be assumed to hold on the line (not only off the zero set), by setting $\left.A\right|_{L}=0$ on $f^{-1}(0)$ and by assuming $p>2$. Therefore we restate it in a simpler form:

$$
\bar{\partial}\left(\left.f\right|_{L}\right)=A_{L} f \text { on } L \cap \Omega, \quad\left\|A_{L}\right\|_{L^{p}(L \cap \Omega)} \leq M
$$

for a constant $M$ independent of $L$.

We now state the main result of Part One:

Theorem A. Let $2<p \leq \infty$. Let $f$ be a continuous function on an open subset $\Omega$ of $\mathrm{C}^{n}$. Assume that for each $j=1, \ldots, n, f$ satisfies $(\mathrm{L})$ for all complex lines $L$ parallel to the $z_{j}$-axis. Then $f^{-1}(0)$ is a complex variety. Moreover, $e^{-u} f$ is holomorphic, for some locally defined $u \in C_{\mathrm{loc}}^{\alpha}$, with $\alpha=1-\frac{2}{p}$ for $2<p<\infty$, and any $\alpha<1$ for $p=\infty$.

Condition (L) plays two roles in the crucial Lemma 3.2: (a) it is used to show that the trivial extension of $A$ is $\bar{\partial}$-closed, (b) once this is proved it is used again for proving that the solution to some $\bar{\partial}$-problem is $C_{\mathrm{loc}}^{\alpha}$-smooth.

To show the analyticity of $f^{-1}(0)$, it suffices to find $u \in L_{\text {loc }}^{\infty}(\Omega)$ solving $\bar{\partial} u=\frac{\bar{\partial} f}{f}$ on $\Omega \backslash f^{-1}(0)$, or on $\Omega$ by extending $\frac{\bar{\partial} f}{f}$ trivially by 0 on $f^{-1}(0)$. There are other ways to insure boundedness, or continuity, or $C_{\text {loc }}^{\alpha}$-smoothness of solutions to the $\bar{\partial}$-problem. This is discussed in details at the beginning of Section 3.

\section{Discussion and an open problem}

The next example shows that the above two results do not hold for $p \leq 2$.

ExAmPLE. Let $a_{k} \in \mathrm{C}^{n}$ with $0<\left|a_{k}\right|<1 / 2$ and $a_{k} \rightarrow 0$ as $k \rightarrow \infty$. Let

$$
f(z)=\frac{1}{(\log |z|) \prod_{k=1}^{\infty}|\log | z-a_{k}||^{\frac{1}{k^{2}}}} .
$$


Then $f$ is continuous on $\mathrm{D}_{1 / 2}^{n}$, and $\bar{\partial}\left(f_{L}\right)=a_{L} f$ with

$$
a_{L}(z)=-\frac{z \cdot d \bar{z}}{2|z|^{2} \log |z|}-\sum \frac{1}{2 k^{2}} \frac{\left(z-a_{k}\right) \cdot d \bar{z}}{\left|z-a_{k}\right|^{2} \log \left|z-a_{k}\right|} \in L^{2}\left(L \cap \mathrm{D}_{1 / 2}^{n}\right) .
$$

Also $\bar{\partial} f=A f$ with $A \in L^{2 n}\left(\mathrm{D}_{1 / 2}^{n}\right)$.

Theorem A is motivated by recent work of Pali [9], who proved, among other results, that $f^{-1}(0)$ is a complex variety under the assumptions that $f$ is a vector of smooth functions on $\Omega \subset C^{n}, \bar{\partial} f=A f$ on $\Omega$ with $A \in C^{\infty}$, and $f$ satisfies a certain finite resolution property. In [9], the difficulties lie in the case when $f$ is not a scalar function.

The following problem remains open:

Problem A. (1) Let $f$ be a vector of continuous functions on a domain $\Omega \subset C^{n}$ satisfying the $\bar{\partial}$-inequality $|\bar{\partial} f| \leq K|f|$ on $\Omega \backslash f^{-1}(0)$. Is $f^{-1}(0)$ a complex variety?

(2) Back to the scalar case: Let $f$ be a continuous function on $\Omega \subset \mathrm{C}^{n}(n>$ 1). Assume that $\bar{\partial} f=A f$ on $\Omega \backslash f^{-1}(0)$ with $A \in L_{(0,1)}^{p}\left(\Omega \backslash f^{-1}(0)\right)$. If $2 n<p<\infty$, is $f^{-1}(0)$ a complex variety?

\section{One dimensional vector-valued case}

Let $\mathrm{D}$ be the unit disc in $\mathrm{C}, \mathrm{D}_{r}$ the disc of radius $r$ centered at the origin, and $\mathrm{D}_{r}(z)$ the disc with radius $r$, centered at $z$. For $f \in L^{p}\left(\mathrm{D}_{\delta}\right)$ with $2<p \leq \infty$, define

$$
T f(z)=\frac{i}{2 \pi} \int_{|\xi|<\delta} \frac{f(\xi)}{z-\xi} d \xi \wedge d \bar{\xi}
$$

Recall the well-known estimates

$$
\begin{aligned}
|T f(z)| & \leq c_{p} \delta^{1-\frac{2}{p}}\|f\|_{L^{p}\left(\mathrm{D}_{\delta}\right)}, \quad 2<p \leq \infty, \\
\left|T f\left(z^{\prime}\right)-T f(z)\right| & \leq c_{p}\|f\|_{L^{p}\left(\mathrm{D}_{\delta}\right)}\left|z^{\prime}-z\right|^{1-\frac{2}{p}}, \quad 2<p<\infty, \\
\left|T f\left(z^{\prime}\right)-T f(z)\right| & \leq c_{p}\|f\|_{L^{\infty}\left(\mathrm{D}_{\delta}\right)}\left|z^{\prime}-z\right|\left(1+|\log \delta|+|\log | z^{\prime}-z||\right),
\end{aligned}
$$

where $c_{p}$ depends only on $p$. Also $\frac{\partial}{\partial \bar{z}} T f=f$ on $\mathbf{D}_{\delta}$ in the distribution sense (see [1]).

The following lemma for the scalar case will be repeatedly used. Note that the lemma gives us Proposition C.

Lemma 2.1. Let $2<p \leq \infty$. Let $\phi_{p}(\delta)=\delta^{1-\frac{2}{p}}$ for $p \neq \infty$ and $\phi_{\infty}(\delta)=$ $\delta(1+|\log \delta|)$. Let $f: \mathrm{D} \rightarrow \mathrm{C}^{m}$ be a continuous map. Assume that for some 
$m \times m$ matrix $A=\left(a_{j k}\right)$ with $a_{j k} \in L^{p}\left(\mathrm{D} \backslash f^{-1}(0)\right)$

$$
\frac{\partial f(z)}{\partial \bar{z}}=A(z) f(z), \quad\|A\|_{L^{p}\left(\mathrm{D} \backslash f^{-1}(0)\right)} \leq M<\infty
$$

holds in the distribution sense on $\mathrm{D} \backslash f^{-1}(0)$. There is a constant $\epsilon>0$ such that if $\delta>0$ and $M \phi_{p}(\delta)<\epsilon$, then for each $z \in \mathrm{D}$, there is an invertible $m \times m$ matrix $I+g$ on $\mathrm{D}_{\delta}(z)$ such that $(I+g) f$ is holomorphic on $\mathrm{D}_{\delta}(z) \cap \mathrm{D}$, and $g \in C^{\alpha}\left(\mathrm{D}_{\delta}(z)\right)$ with $\alpha=1-\frac{2}{p}$ for $2<p<\infty$ and any $\alpha<1$ for $p=\infty$. Moreover, $\sup _{w \in \mathrm{D}_{\delta}(z)}|g(w)| \leq c_{p, m} M \phi_{p}(\delta)$ for some constant $c_{p, m}$, and $\frac{\partial f}{\partial \bar{z}}=\tilde{A} f$ on $\mathrm{D}$ where $\tilde{A}$ equals $A$ on $\mathrm{D} \backslash f^{-1}(0)$ and zero on $f^{-1}(0)$.

Proof. Set $\tilde{A}=A$ on $\mathrm{D} \backslash f^{-1}(0)$ and $\tilde{A}=0$ on $f^{-1}(0) \cup(\mathrm{C} \backslash \mathrm{D})$. Still denote $\tilde{A}$ by $A$. We may assume that $z=0$. We need

$$
0=\frac{\partial}{\partial \bar{z}}((I+g) f)=\left(\frac{\partial}{\partial \bar{z}} g+(I+g) A\right) f
$$

on $\mathrm{D}_{\delta}$. For a matrix $u$ of functions in $L^{p}\left(\mathrm{D}_{\delta}\right)$, define

$$
T u(z)=\frac{i}{2 \pi} \int_{|\xi|<\delta} \frac{u(\xi)}{z-\xi} d \xi \wedge d \bar{\xi},
$$

where the integration takes place entry by entry. The $\delta$, depending only on $m$ and $p$, will be determined later.

Consider the equation

$$
g+T((I+g) A)=0 .
$$

For the matrices $g$ and $A=\left(a_{j k}\right)$, put

$$
\|g\|=\sup _{j, k, \mathbf{D}_{\delta}}\left\{\left|g_{j k}(z)\right|\right\}, \quad\|A\|_{p}=\max _{j, k}\left\{\left\|a_{j k}\right\|_{L^{p}\left(\mathbf{D}_{\delta}\right)}\right\} .
$$

Recall that $T((I+g) A)$ is continuous on $\overline{\mathrm{D}}_{\delta}$, if $g$ is continuous on $\overline{\mathrm{D}}_{\delta}$. We also have

$$
\begin{aligned}
\left\|T\left(\left(I+g_{2}\right) A\right)-T\left(\left(I+g_{1}\right) A\right)\right\| & \leq c_{p, m} \phi_{p}(\delta)\|A\|_{p} \cdot\left\|g_{2}-g_{1}\right\|, \\
\|T(A)\| & \leq c_{p, m} \phi_{p}(\delta)\|A\|_{p} .
\end{aligned}
$$

Therefore, $g=\lim _{k \rightarrow \infty} S^{k}(0)$, where $S(g)=-T((I+g) A)$, is the unique matrix that is continuous on $\overline{\mathrm{D}}_{\delta}$ and satisfies $(*)$ on $\mathrm{D}_{\delta}$, provided

$$
M \phi_{p}(\delta)<\frac{1}{2 c_{p, m}} .
$$


Moreover, the $g$ satisfies

$$
\|g\| \leq \frac{c_{p, m}\|A\|_{p} \phi_{p}(\delta)}{1-c_{p, m}\|A\|_{p} \phi_{p}(\delta)} \leq 2 c_{p, m} M \phi_{p}(\delta) .
$$

By $(*),(I+g) f$ is holomorphic on $\mathrm{D}_{\delta} \backslash f^{-1}(0)$. Let $h$ be an entry of $(I+g) f$. We know that $h$ is continuous on $\mathbf{D}_{\delta}$. If $h$ does not vanish at $z_{0} \in \mathbf{D}_{\delta}$, then $f\left(z_{0}\right) \neq 0$ either. Hence $h$ is holomorphic near $z_{0}$. By Rado's theorem, $h$ is holomorphic on $\mathrm{D}_{\delta}$. Since $g=-T((I+g) A)$ and $(I+g) A$ is in $L_{\mathrm{loc}}^{p}$ then $g \in C_{\text {loc }}^{\alpha}$ for $\alpha=1-\frac{2}{p}$ when $2<p<\infty$ and any $\alpha<1$ for $p=\infty$. on D.

Since $f$ is identically zero or has isolated zeros, it is easy to see that $\frac{\partial f}{\partial \bar{z}}=\tilde{A} f$

When $f$ is scalar, one simply takes the above $1+g$ to be the so-called integrating factor, $e^{-u}$, where $u=A * \frac{1}{\pi z}$ by setting $A=0$ on $f^{-1}(0)$. On the other hand, the above contraction argument avoids the Rado theorem, if one has a stronger condition that $\frac{\partial f}{\partial \bar{z}}=A f$ on $D$.

The zero set $f^{-1}(0)$ for the one-dimensional case is studied by IvashkovichShevchishin [7], under the assumption that $\bar{\partial} f=A f$ on the whole domain $\Omega \subset$ C. See also Floer-Hofer-Salamon [4].

\section{Proof of Theorem A (scalar-valued case, functions defined on $\mathrm{C}^{n}$ )}

The heart of the matter is to prove that $B$, the trivial extension of $\frac{\bar{\partial} f}{f}$, is $\bar{\partial}$-closed. This is done in Lemma 3.2, but we start in Lemma 3.1 with some remarks that will allow us later to prove that the solutions to $\bar{\partial} u=B$ are locally bounded.

\subsection{Restriction of $\bar{\partial} f=A f$ on lines}

First we need to discuss the restriction of $\bar{\partial} f=A f$ on complex lines.

However, the reader could skip this section 3.1 (remarks (a)-(d) below) and go directly to section 3.2 (beginning of the proof of Theorem A), by first accepting the assertion: if $f$ satisfies condition (L) for almost all $z_{j}$-lines and if $B$, the trivial extension of $\frac{\bar{\partial} f}{f}$ by 0 on $f^{-1}(0)$, is $\bar{\partial}$-closed, then all solutions $u$ to $\bar{\partial} u=B$ are locally bounded on $\Omega$. The latter will suffice for the proof of analyticity of $f^{-1}(0)$.

Let $f$ be a continuous function defined on an open subset $D$ of $\mathrm{C}^{n}$. It is worth pointing out the following.

(a) Let $1<p \leq \infty$. Let $M$ be a positive constant. Assume that for almost all $z_{1}$-lines $L$

$$
\frac{\left.\partial f\right|_{L}}{\partial \bar{z}_{1}}=A_{L} f, \quad\left\|A_{L}\right\|_{L^{p}(L \cap D)} \leq M .
$$


Then in the distribution sense

$$
\frac{\partial f}{\partial \bar{z}_{1}}=B f
$$

on $D$, for some function $B \in L_{\mathrm{loc}}^{p}(D)$, that we set to be 0 on $f^{-1}(0)$.

That function $B$ satisfies:

$$
\|B\|_{L^{p}\left(D \cap L_{\epsilon}\right)} \leq\left(\pi \epsilon^{2}\right)^{\frac{n-1}{p}} M,
$$

where $L_{\epsilon}$ is the $\epsilon$-neighborhood of $L$ in $C^{n}$ equipped with the sup-norm. (In the latter formulation we avoid the problem of having to restrict $B$ to lines.)

Proof. Check $\frac{\partial f}{\partial \bar{z}_{1}}=B f$ for some $B \in L_{\text {loc }}^{p}(D)$. Fix a compact subset $K$ of $D$. Let $\varphi$ be a smooth function whose support is contained in $K$. Put $z^{\prime}=\left(z_{2}, \ldots, z_{n}\right)$ and

$$
\begin{aligned}
& d V\left(z^{\prime}\right)=\left(\frac{i}{2}\right)^{n-1} d z_{2} \wedge d \bar{z}_{2} \wedge \cdots \wedge d z_{n} \wedge d \bar{z}_{n}, \\
& d V(z)=\frac{i}{2} d z_{1} \wedge d \bar{z}_{1} \wedge d V\left(z^{\prime}\right) .
\end{aligned}
$$

Then

$$
\begin{aligned}
& \left|\left(\frac{\partial f}{\partial \bar{z}_{1}}, \varphi\right)\right| \\
& =\left|\iint f \frac{\partial \varphi}{\partial \bar{z}_{1}} \frac{i}{2} d z_{1} \wedge d \bar{z}_{1} \wedge d V\left(z^{\prime}\right)\right| \\
& =\left|\int\left(\frac{\left.\partial f\right|_{z^{\prime}} \text { fixed }}{\partial \bar{z}_{1}}, \varphi\right)_{z_{1}} d V\left(z^{\prime}\right)\right| \\
& =\left|\int\left(\left.\int A\right|_{L\left(z^{\prime}\right)} f \varphi \frac{i}{2} d z_{1} \wedge d \bar{z}_{1}\right) d V\left(z^{\prime}\right)\right|, \quad L\left(z^{\prime}\right)=\left\{\left(z_{1}, z^{\prime}\right): z_{1} \in \mathrm{C}\right\} \cap D \\
& \text { Hölder } M \int\|f \varphi\|_{L^{q}\left(z_{1}\right)} d V\left(z^{\prime}\right) \\
& \underset{\text { Hölder }}{\leq} M\left|K^{\prime}\right|^{\frac{1}{p}}\left[\int|f \varphi|^{q} \frac{i}{2} d z_{1} \wedge d \bar{z}_{1} \wedge d V\left(z^{\prime}\right)\right]^{\frac{1}{q}},
\end{aligned}
$$

where $K^{\prime}$ is the projection of $K$ by $z \rightarrow\left(z_{2}, \ldots, z_{n}\right)$ and $\left|K^{\prime}\right|=\int_{K^{\prime}} d V\left(z^{\prime}\right)$. So $\left|\left(\frac{\partial f}{\partial \bar{z}_{1}}, \varphi\right)\right| \leq M\left|K^{\prime}\right|^{\frac{1}{p}}\|f \varphi\|_{L^{q}}$, i.e $f \varphi \rightarrow\left(\frac{\partial f}{\partial \bar{z}_{1}}, \varphi\right)$ is continuous in $L^{q}$-norm. 
So $\left(\frac{\partial f}{\partial \bar{z}_{1}}, \varphi\right)=\int B f \varphi d V(z)$ for any $\varphi$ supported in $K$, for some $B$ supported in $K$, vanishing on $f^{-1}(0)$, and satisfying

$$
\|B\|_{L^{p}} \leq M\left|K^{\prime}\right|^{\frac{1}{p}} .
$$

For the general case, let $\left\{\chi_{k}\right\}$ be a partition of unity for a locally finite open covering $\left\{U_{j}\right\}$ of $D$. Then $\frac{\partial f}{\partial \bar{z}_{1}}=B_{k} f$ on $U_{k}$, and $\left(\frac{\partial f}{\partial \bar{z}_{1}}, \varphi\right)=\sum\left(\frac{\partial f}{\partial \bar{z}_{1}}, \chi_{k} \varphi\right)=$ $(B, \varphi)$ for $B=\sum \chi_{k} B_{k} \in L_{\text {loc }}^{p}$. Finally, (E) comes from (3.1) and the uniqueness of $B$ (up to a set of measure 0 and requiring $\left.B\right|_{f^{-1}(0)}=0$ ) since $f$ is continuous.

(b) Let $1<p \leq \infty$. Assume that $\frac{\partial f}{\partial \bar{z}_{1}}=B f$ on $D$ with $B$ satisfying (E) for all $z_{1}$-lines $L$ and all $\epsilon>0$. Then (L) holds for all $z_{1}$-lines $L$. In other words, when $f$ is continuous, (L) and (E) are equivalent. Moreover, that (L) holds for almost all $z_{1}$-lines implies (L) for all $z_{1}$-lines.

Proof. Let $\chi \geq 0$ be a smooth function on $C^{n}$ with compact support and $\int \chi(z) d V(z)=1$. Put $\chi_{\delta}(z)=\frac{1}{\delta^{2 n}} \chi(\delta z)$ and $B_{\delta}=B * \chi_{\delta}$. We may assume that $L$ is the $z_{1}$-axis. Fix a compact subset $K$ of $L \equiv L \cap D \subset \mathrm{C}$. Then (3.2)

$$
\begin{aligned}
\left\|B_{\delta}\right\|_{L^{p}\left(K \times \mathrm{D}_{\epsilon}^{n-1}\right)} & =\left(\int_{K \times \mathrm{D}_{\epsilon}^{n-1}}\left|\int B(z-\delta w) \chi(w) d V(w)\right|^{p} d V(z)\right)^{\frac{1}{p}} \\
& \leq \int\left(\int_{K \times \mathrm{D}_{\epsilon}^{n-1}}|B(z-\delta w)|^{p} d V(z)\right)^{\frac{1}{p}} \chi(w) d V(w) \\
& \leq \int\left(\int_{D \cap C \times\left(-\delta w^{\prime}+\mathrm{D}_{\epsilon}^{n-1}\right)}|B(z)|^{p} d V(z)\right)^{\frac{1}{p}} \chi(w) d V(w) \\
& \leq\left(\pi \epsilon^{2}\right)^{\frac{n-1}{p}} M, \quad w=\left(w_{1}, w^{\prime}\right) .
\end{aligned}
$$

Thus, $\left\|\left.B_{\delta}\right|_{K \times\left\{0^{\prime}\right\}}\right\|_{L^{p}} \leq M$, since $B_{\delta}$ is continuous. For each smooth function $\varphi$ with compact support in $L$,

$$
\left(\frac{\left.\partial f\right|_{L}}{\partial \bar{z}_{1}}, \varphi\right)=\lim _{\delta \rightarrow 0}\left(\frac{\left.\partial f_{\delta}\right|_{L}}{\partial \bar{z}_{1}}, \varphi\right)=\lim _{\delta \rightarrow 0}\left(\left.(B f)_{\delta}\right|_{L}, \varphi\right)=\lim _{\delta \rightarrow 0}\left(\left.B_{\delta}\right|_{L} f, \varphi\right) .
$$

For small $\delta>0$

$$
\begin{aligned}
\left\|B_{\delta}\left(\cdot, z^{\prime}\right)\right\|_{L^{p}(K)} & =\left(\int_{K}\left|\int B\left(\left(z_{1}, z^{\prime}\right)-\delta w\right) \chi(w) d V(w)\right|^{p} \frac{i}{2} d z_{1} \wedge d \bar{z}_{1}\right)^{\frac{1}{p}} \\
& \leq M
\end{aligned}
$$


Using the weak compactness in $L^{p}$, we find a sequence $\left.B_{\delta_{k}}\right|_{L}$ that converges weakly to some $A_{L} \in L^{p}(L)$ with $\left\|A_{L}\right\|_{L^{p}} \leq M$.

By Proposition C, remarks a) and b), we know that the condition

$$
\bar{\partial} f=A f \text { on } \Omega \backslash f^{-1}(0), \quad A \in L^{\infty}\left(\Omega \backslash f^{-1}(0)\right)
$$

is equivalent to

$$
\bar{\partial} f=A f \text { on } \Omega, \quad A \in L^{\infty}(\Omega),
$$

in which $A$ is set to be 0 on $f^{-1}(0)$.

(c) Let $v=v_{1} d \bar{z}_{1}+\cdots+v_{n} d \bar{z}_{n}$ with $v_{j} \in L_{\text {loc }}^{1}(\Omega)$ be $\bar{\partial}$-closed on $D$. The local existence and boundedness of solutions $u$ to $\bar{\partial} u=v$ follow from any of the following conditions:

(i) The integral of $\int_{D} \frac{|v(w)|}{|z-w|^{2 n-1}} d V(w)$ is bounded (independently of $z$ ).

(ii) For each $v_{j}$ there is $k$ such that for some $p>2$ and $M>0$,

$\left\|v_{j}\right\|_{L^{p}(D \cap L)} \leq M$ for (almost) all $z_{k}$-lines $L$.

(iii) $v$ is in $L^{p}(D)$ for some $p>2 n$.

In fact, all local solutions are continuous for case (ii), and are in $C_{\text {loc }}^{\alpha}$ with $\alpha=1-\frac{2 n}{p}$ for $2 n<p<\infty$ and any $\alpha<1$ for $p=\infty$, for (iii).

Proof. We may assume that $D=\mathrm{D}^{n}$. It is elementary that (iii) implies (i). (ii) implies (i) too: Assume that $\left\|v_{j}\right\|_{L^{p}(D \cap L)} \leq M$ for all $z_{k}$-lines $L$ (say $k=n)$. Then

$$
\begin{aligned}
& \int_{D} \frac{\left|v_{j}(w)\right|}{|z-w|^{2 n-1}} d V(w) \\
& \quad \leq M \int_{|z|<1}\left(\int_{\left|z_{n}\right|<1} \frac{1}{\left(|' z|^{2}+\left|z_{n}\right|^{2}\right)^{(2 n-1) q / 2}} \frac{i}{2} d z_{n} \wedge d \bar{z}_{n}\right)^{\frac{1}{q}} d V\left(^{\prime} z\right) \\
& \quad \leq c M \int_{|z|<1} 1+\left.||^{\prime} z\right|^{\frac{2}{q}-(2 n-1)} d V\left(^{\prime} z\right) \\
& \quad=\tilde{c} M \int_{0}^{1} r^{2 n-3}+r^{-\frac{2}{p}} d r<\infty
\end{aligned}
$$

for $\frac{1}{q}=1-\frac{1}{p}$ (and $\left.n>1\right)$.

Assume that (i) holds with $D$ being the unit ball and $\bar{\partial} v=0$. 
Recall the Bochner-Martinelli kernels:

$$
\begin{aligned}
\frac{(n-1) !}{(2 \pi i)^{n}} \sum_{j}(-1)^{j-1} \frac{\bar{\zeta}_{j}-\bar{z}_{j}}{|\zeta-z|^{2 n}} \wedge_{k \neq j}\left(d \bar{\zeta}_{k}-d \bar{z}_{k}\right) \wedge d \zeta_{1} \wedge \cdots & \wedge d \zeta_{n} \\
& =\sum_{q=0}^{n-1} \Omega_{q}(z, \zeta)
\end{aligned}
$$

where $\Omega_{q}$ is of bidegree $(n, n-q-1)$ in $\zeta$ and bidegree $(0, q)$ in $z$. For a $(0, q)$-form $u$ on $D$ with $q>0$, define

$$
B_{M} * u(z)=-\int_{\zeta \in D} u(\zeta) \wedge \Omega_{q-1}(z, \zeta) .
$$

By the Koppelman formula ([5], p. 57), $w=\bar{\partial}\left(B_{M} * w\right)-B_{M} * \bar{\partial} w$, if $w$ is a smooth $(0,1)$-form on $D$ with compact support.

Let $v_{\epsilon}=\sum_{j=1}^{n}\left(v_{j}\right)_{\epsilon} d \bar{z}_{j}$, where the smoothing $\left(v_{j}\right)_{\epsilon}$ is defined in the proof of remark b). Let $\chi$ be a smooth function supported in the ball $D_{1 / 2}:|z|<1 / 2$. For small and positive $\epsilon, v_{\epsilon}$ is well-defined and $\bar{\partial}$-closed on $D_{1 / 2}$. Thus

$$
\chi v_{\epsilon}=\bar{\partial}\left(B_{M} *\left(\chi v_{\epsilon}\right)\right)-B_{M} *\left(\bar{\partial} \chi \wedge v_{\epsilon}\right), \quad \text { on } \quad D
$$

By the Fubini theorem, $\left\|B_{M} *\left(\chi v_{\epsilon}\right)\right\|_{L^{\infty}\left(D_{1 / 2}\right)}+\left\|B_{M} *\left(\bar{\partial} \chi \wedge v_{\epsilon}\right)\right\|_{L^{\infty}\left(D_{1 / 2}\right)} \leq$ $c\left\||v| * \frac{1}{|z|^{2 n-1}}\right\|_{L^{\infty}(D)}$ for some constant $c$. On $\bar{D}_{1 / 3}, B_{M} *\left(\bar{\partial} \chi \wedge v_{\epsilon}\right)$ is smooth and $\bar{\partial}$-closed for small $\epsilon>0$. It is well-known that there is a smooth function $\tilde{u}_{\epsilon}$ such that $\bar{\partial} \tilde{u}_{\epsilon}=B_{M} *\left(\bar{\partial} \chi \wedge v_{\epsilon}\right)$ and $\left\|\tilde{u}_{\epsilon}\right\|_{L^{\infty}\left(D_{1 / 3}\right)} \leq \tilde{c}\left\|B_{M} *\left(\bar{\partial} \chi \wedge v_{\epsilon}\right)\right\|_{L^{\infty}\left(D_{1 / 3}\right)}$. We now have $\bar{\partial}\left(B_{M} *\left(\chi v_{\epsilon}\right)-\tilde{u}_{\epsilon}\right)=v_{\epsilon}$ on $D_{1 / 3}$. By the weak compactness, the week-limit $u$ of some sequence $B_{M} *\left(\chi v_{\epsilon_{k}}\right)-\tilde{u}_{\epsilon_{k}}$ is bounded and satisfies $\bar{\partial} u=v$.

For (ii), the continuity of $u$ follows from the continuity of $\left|v_{j}\right| * \frac{1}{|z|^{2 n-1}}$. For (iii), it is elementary that $B_{M} * v \in C_{\mathrm{loc}}^{\alpha}$ when $v \in L^{p}(D)$ and $p>2 n$.

For a complex line $L$ in $\mathrm{C}^{n}$, denote by $L_{\epsilon}$ the $\epsilon$-neighborhood of $L$ in $C^{n}$ with the sup-norm.

We now point out a special case, which is actually used in our proofs.

(d) Let $2<p \leq \infty$. Let $u \in L_{\text {loc }}^{1}(D)$ and $\bar{\partial} u=v_{1} d \bar{z}_{1}+\cdots+v_{n} d \bar{z}_{n}$. Assume that for each $j$ there is a constant $M$ such that

$$
\left\|v_{j}\right\|_{L^{p}\left(D \cap L_{j, \epsilon}\right)} \leq M\left(\pi \epsilon^{2}\right)^{\frac{n-1}{p}}
$$

for all $z_{j}$-lines $L_{j}$ and all $\epsilon>0$, then $u \in C_{\text {loc }}^{\alpha}$ for $\alpha=1-\frac{2}{p}(2<p<\infty)$ or for all $\alpha<1(p=\infty)$. 
Proof. By (c) (ii), we know that all local solutions $u$ are continuous. Repeat the argument in (b) for $\frac{\partial u}{\partial \bar{z}_{j}}=v_{j}$ (in a simpler way). We have $\frac{\left.\partial u\right|_{L}}{\partial \bar{z}_{j}}=\tilde{v}_{L}$ with $\left\|\tilde{v}_{L}\right\|_{L^{p}} \leq M$. Now the local Hölder- $\alpha$ norm of $\left.u\right|_{L}$ is bounded by some constant independent of $L$. Therefore $\left.u\right|_{L}$ (and hence $u$ ) is $C_{\mathrm{loc}}^{\alpha}$-smooth, by the well-known estimates stated at the beginning of Section 2.

\section{2. $\bar{\partial}$-closedness of trivial extension of $\frac{\bar{\partial} f}{f}$, when $f^{-1}(0)$ is the graph of a continuous function}

As already said, the heart of the matter is to prove that $B$, the trivial extension of $\frac{\bar{\partial} f}{f}$, is $\bar{\partial}$-closed. Once this is done the existence of an integrating factor and hence the analyticity of $f^{-1}(0)$ is trivial. However the proof goes as follows. In this section 3.2 we prove $\bar{\partial}$-closedness of $B$ near specific points of $f^{-1}(0)$ (Lemma 3.2). We then get the analyticity of $f^{-1}(0)$ off an exceptional set (in the notations below, the set where $N(p)=\infty$ ). Using induction on dimension, the proof of $\bar{\partial}$-closedness of $B$ is then achieved in section 3.3.

By our assumptions, if $f$ vanishes at $p$, then for any complex line $L$ passing through $p$ and parallel to a coordinate axis, there is a continuous function $u$ so that $\left.e^{-u} f\right|_{L}$ is holomorphic on $L \cap \Omega$. Define the order of vanishing of $\left.f\right|_{L}$ at $p$ to be the vanishing order of $\left.e^{-u} f\right|_{L}$ at $p$. We also define the number of zeros of $\left.f\right|_{L}$ to be the number of zeros of $\left.e^{-u} f\right|_{L}$. Both are independent of the choice of $u$.

We start with the following.

Lemma 3.1. Let $2<p \leq \infty$. Let $f(z)$ be a continuous function on $\mathrm{D}^{n}$. Assume that there exists a positive constant $M$ such that for all complex lines $L$ parallel to the $z_{n}$-axis, $\bar{\partial}\left(\left.f\right|_{L}\right)=A_{L} f$ on $\mathrm{D}^{n} \cap L$ and $\left\|A_{L}\right\|_{L^{p}\left(L \cap \mathrm{D}^{n}\right)} \leq M$. Assume that $f(0)=0$ and $f\left(0, z_{n}\right)$ does not vanish when $0<\left|z_{n}\right| \leq \epsilon_{0}<1$.

(i) There exists $\delta>0$ such that for each' $z \in \mathrm{D}_{\delta}^{n-1}, f\left(' z, z_{n}\right)$ has exactly $N$ zeros $z_{n}=r_{j}(' z), j=1, \ldots, N$ in the disc $\left\{z_{n}:\left|z_{n}\right|<\epsilon_{0}\right\}$, counting multiplicity. Moreover, for each $\epsilon \in\left(0, \epsilon_{0}\right)$ if the $\delta$ is sufficiently small we have $\left|r_{j}(z)\right|<\epsilon$ for $|' z|<\delta$ and $\lim _{z \rightarrow 0} r_{j}(' z)=0$ for all $j$.

(ii) If the above $r_{j}$ are all the same, denoted by $r$, then $f(z)=\left(\left(z_{n}-\right.\right.$ $r(z)) v(z))^{N}$, where $v$ is continuous on $\mathbf{D}_{\delta}^{n-1} \times \mathrm{D}_{\epsilon_{0}} \backslash f^{-1}(0)$, and $v, \frac{1}{v}$ are locally bounded on $\mathrm{D}_{\delta}^{n-1} \times \mathrm{D}_{\epsilon_{0}}$.

Proof. (i) By Proposition C, we know that there exists $0<\epsilon_{1} \leq \epsilon_{0}$ such that for each $\epsilon \in\left(0, \epsilon_{1}\right)$ there exists a function $g(z)$ such that $(1+g(z)) f(z)$ is holomorphic in $z_{n}$ and $|g(z)|<1 / 4$ for $z \in \mathrm{D}^{n-1} \times \mathrm{D}_{\epsilon}$. By definition the number of zeroes of $f$ as a function of $z_{n}$ is the same as the number of zeroes of the holomorphic function $(1+g) f$. So it is the winding number of 
$(1+g) f$ and hence that of $f$, which is constant (in a neighborhood of 0 ). The last conclusion follows from the continuity of $f$ also.

(ii) We reduce it to case $N=1$ first. Choose a continuous root $b(z)=$ $f(z)^{1 / N}$ on $\mathrm{D}_{\delta}^{n-1} \times \frac{\epsilon_{0}}{2}$. On each' $z \times \mathrm{D}_{\epsilon_{0}}$ we find a continuous root $f(z)^{1 / N}$ that agrees with $b(z)$ at $z=\left({ }^{\prime} z, \frac{\epsilon_{0}}{2}\right)$. Thus $f(z)^{1 / N}$ is continuous on $\mathbf{D}_{\delta}^{n-1} \times \mathbf{D}_{\epsilon_{0}}$. Off the zero set of $f^{1 / N}$, we have $\frac{\bar{\partial} f^{1 / N}}{f^{1 / N}}=\bar{\partial} \log f^{1 / N}=\frac{1}{N} \frac{\bar{\partial} f}{f}$.

Assume that $N=1$. The local boundedness of $v$ follows from the above estimate $|g|<1 / 4$. Now

$$
\frac{1}{v}=(1+g(z)) \frac{z_{n}-r(' z)}{(1+g(z)) f(z)}
$$

is also locally bounded, by applying the maximal principle to the holomorphic function $\frac{z_{n}-r(z)}{(1+g(z)) f(z)}$.

REMARKS. (i) We are not claiming that the above $v$ (for $r_{1}=\cdots=r_{N}$ ) is continuous in $z$, though this holds eventually. In fact if $v$ is continuous and if additionally $\bar{\partial}\left(\left.f\right|_{L}\right)=A_{L} f$ on $L \cap \mathrm{D}^{n}$ with $\left\|A_{L}\right\|_{L^{p}\left(L \cap \mathrm{D}^{n}\right)} \leq M$ for all complex lines $L$, then one can conclude that $r$ is holomorphic. To see that, consider the line $L=\{a+t b: t \in \mathrm{C}\}$ where $a=\left(a^{\prime}, a_{n}\right)$ is a zero of $f$ with $\left|a^{\prime}\right|<\delta$ and $b$ is a non-zero vector in $C^{n}$. We know that there is a continuous function $u$ on $L$ such that $e^{-u} f^{1 / N}=v e^{-u}\left(z_{n}-r(z)\right)$ is holomorphic on $L$. Thus $\lim _{t \rightarrow 0} \frac{\left(a_{n}+t b_{n}-r\left(a^{\prime}+t b^{\prime}\right)\right) v(a+t b)}{t}$ exists. Since $a_{n}=r\left(a^{\prime}\right)$ and $v(a) \neq 0$, then $\lim _{t \rightarrow 0} \frac{r\left(a^{\prime}+t b^{\prime}\right)-r\left(a^{\prime}\right)}{t}$ exists for all $b^{\prime}$.

(ii) Let $p>2$. Assume that $f$ is continuous on $\mathrm{D}^{n}$ and $C^{1}$-smooth on $\overline{\mathrm{D}}^{n} \backslash f^{-1}(0)$ and $\frac{\partial f}{\partial \bar{z}_{n}}=a_{L} f$ on $L \cap \mathrm{D}^{n}$ with $\left\|a_{L}\right\|_{L^{p}\left(L \cap \mathrm{D}^{n}\right)} \leq M$ for all $z_{n}$-lines $L$. Then the $v$ (for $r_{1}=\cdots=r_{N}$ ) is continuous. To see that, we let $L=L(z)$ be the $z_{n}$-line passing through $z$. Assuming $N=1$, put $u=A_{L} * \frac{1}{\pi z_{n}}$, where the convolution is on $\left|z_{n}\right|<1$. We already know that $r$ is continuous. Fix $w \in \mathrm{D}^{n}$. We want to show that $u$ is continuous at $w=\left({ }^{\prime} w, w_{n}\right)$. Split $a_{L} * \frac{1}{\pi z_{n}}$ into two parts:

$$
\begin{aligned}
u(z)=\frac{i}{2 \pi} \int_{|\zeta-r(' w)|<\delta,|\zeta|<1} & \frac{a_{L}(\zeta)}{z_{n}-\zeta} d \zeta \wedge d \bar{\zeta} \\
& \quad+\frac{i}{2 \pi} \int_{|\zeta-r(' w)|>\delta,|\zeta|<1} \frac{\frac{\partial f}{\partial \bar{\zeta}}\left({ }^{\prime} z, \zeta\right)}{\left(z_{n}-\zeta\right) f(' z, \zeta)} d \zeta \wedge d \bar{\zeta} .
\end{aligned}
$$

Since $\frac{1}{z} \in L_{\mathrm{loc}}^{q}$ for $1 \leq q<2$, the first term tends to 0 as $\delta \rightarrow 0$. For a fixed $\delta$, the second term is continuous in $z$ at $w$. Thus $u$ is continuous on $\mathbf{D}^{n}$. As before by Rado's theorem $e^{-u} f$ is holomorphic in $z_{n}$. Since $e^{-u} v=\frac{e^{-u} f}{z_{n}-r(z)}$ is 
holomorphic in $z_{n}$, then

$$
\left(e^{-u} v\right)(z)=\frac{1}{2 \pi i} \int_{|\zeta|=\epsilon_{0}} \frac{e^{\left.-u{ }^{\prime} z, \zeta\right)} f\left({ }^{\prime} z, \zeta\right)}{(\zeta-r(' z))\left(\zeta-z_{n}\right)} d \zeta, \quad\left|z_{n}\right|<\epsilon_{0} .
$$

Thus $e^{-u} v$ and hence $v$ is continuous.

Lemma 3.2. Let $2<p \leq \infty$. Let $f(z)$ be a continuous function on $\mathrm{D}^{n}$, satisfying the condition $(\mathrm{L})$ (with $\Omega=\mathrm{D}^{n}$ ). Assume that for each $z \in \mathrm{D}^{n-1}$, $f\left(' z, z_{n}\right)$ has exactly one zero in $z_{n}=r(' z) \in \mathrm{D}$, and its multiplicity is 1 . Then $B$, the trivial extension of $\frac{\bar{\partial} f}{f}$ by 0 on $f^{-1}(0)$, is $\bar{\partial}$-closed in the sense of distribution on $\mathrm{D}^{n}$, and $r$ is holomorphic.

Proof. $A=\frac{\bar{\partial} f}{f}$ is obviously $\bar{\partial}$-closed on the complement of $f^{-1}(0)$. The main point in the proof consists in proving that its trivial extension, by 0 on $f^{-1}(0)$, is $\bar{\partial}$-closed on $\mathrm{D}^{n}$ (a problem that of course does not exist for $n=1$ ). In case $\frac{\bar{\partial} f}{f}$ is bounded, this is straightforward if one assumes (but we don't) that $f^{-1}(0)$ has a basis of neighborhoods whose boundaries have $(2 n-1)$ Hausdorff measure tending to 0 . The trivial extension of $A=\frac{\bar{\partial} f}{f}$ is precisely the $B \in L_{\text {loc }}^{p}\left(\mathrm{D}^{n}\right)$ in remark 3a). So $\bar{\partial} f=B f$ on $\mathrm{D}^{n}$ (not just on $\mathrm{D}^{n} \backslash f^{-1}(0)$ ).

Return to our setting. We want to show that $\bar{\partial} B=0$ near 0 . Let $d_{v}(z)$ be the vertical distance from $z$ to the graph $z_{n}=r(' z)$. So $d_{v}(z)=\left|z_{n}-r(' z)\right|$. Let $d(z)$ be the Euclidean distance from $z$ to the graph. We will prove that $\epsilon$-neighborhoods of the graph $z_{n}=r\left({ }^{\prime} z\right)$ with respect to $d$ and $d_{v}$ respectively have comparable volumes. This is achieved by comparing $d(z), d_{v}(z)$ with $|f(z)|$.

If $\tilde{f}(z)=f(\epsilon z)$ and $\frac{\partial f}{\partial \bar{z}_{n}}=a f$ (the one-dimensional distribution derivative off $f^{-1}(0)$ with $z_{1}, \ldots, z_{n-1}$ being fixed first) we have $\frac{\partial \tilde{f}}{\partial \bar{z}_{n}}=\tilde{a} \tilde{f}$ off $\tilde{f}^{-1}(0)$ with

$$
\begin{aligned}
& \left(\int_{\left|z_{n}\right|<1, \tilde{f}(z) \neq 0}|\tilde{a}|^{p} \frac{i}{2} d z_{n} \wedge d \bar{z}_{n}\right)^{\frac{1}{p}} \\
& =\left(\int_{\left|z_{n}\right|<1, f(z) \neq 0}|\epsilon a(\epsilon z)|^{p} \frac{i}{2} d z_{n} \wedge d \bar{z}_{n}\right)^{\frac{1}{p}} \\
& =\epsilon^{1-\frac{2}{p}}\left(\int_{\left|z_{n}\right|<\epsilon, f(z) \neq 0}\left|a\left(\epsilon z^{\prime}, z_{n}\right)\right|^{p} \frac{i}{2} d z_{n} \wedge d \bar{z}_{n}\right)^{\frac{1}{p}} .
\end{aligned}
$$

Since $p>2$, by a dilation, we may assume that the $L^{p}$ norms of $A_{L}$ on $L \cap \mathbf{D}^{n} \backslash f^{-1}(0)$ are small on all $z_{j}$-lines $L$. On each $z_{j}$-line $L$, there is a continuous function $g$ such that $(1+g) f$ is holomorphic on $L \cap \mathbf{D}^{n}$ and $|g|<1 / 4$. We may also assume that $|f|<1$. 
Let $f(0)=0$. Let $z \in \mathrm{D}_{1 / 4}^{n}$ with $f(z) \neq 0$.

To compare $f(z)$ with $d_{v}(z)$, recall from Lemma 3.1(ii) that

$$
f(z)=\left(z_{n}-r\left({ }^{\prime} z\right)\right) v(z), \quad z \in \mathrm{D}^{n},
$$

where $\frac{1}{v}$ is locally bounded. Restricting to a smaller polydisc if necessary, we may assume $|v|>1 / c$ on $\mathrm{D}^{n}$ for a fixed constant $c>0$. Thus $d_{v}(z)=$ $\left|z_{n}-r(' z)\right| \leq c|f(z)|$.

To compare $f(z)$ with $d(z)$ take $z^{*} \in f^{-1}(0) \cap \overline{\mathrm{D}}_{1 / 2}^{n}$ such that $d(z)=\left|z-z^{*}\right|$. Let $z^{*}=\left(z_{1}^{*}, \ldots, z_{n}^{*}\right), z=\left(z_{1}, \ldots, z_{n}\right)$, and $w_{j}=\left(z_{1}, \ldots, z_{j-1}, z_{j}^{*}, \ldots, z_{n}^{*}\right)$. Start with $w_{1} \in f^{-1}(0)$ and connect $w_{j}$ to $w_{j+1}$ by a $z_{j}$-line $L_{j}$. We want to show that $\left|f\left(w_{j}\right)\right| \leq 8(j-1) 2^{j} d(z)$. The inequality is trivial for $j=1$. Assume the inequality holds for $f\left(w_{k}\right)$. We find a function $g,|g|<1 / 4$, such that $(1+g) f$ is holomorphic on $L_{k} \cap \mathrm{D}^{n}$. By the maximum principle, we have

$$
\left|((1+g) f)\left(w_{k+1}\right)-((1+g) f)\left(w_{k}\right)\right| \leq 8\left|w_{k+1}-w_{k}\right| \leq 8 d(z) .
$$

Hence $\left|f\left(w_{k+1}\right)\right| \leq 2\left|f\left(w_{k}\right)\right|+16 d(z) \leq 8 k 2^{k+1} d(z)$. Therefore $|f(z)| \leq$ $8 n 2^{n} d(z)$. Thus

$$
d(z) \leq d_{v}(z) \leq c_{1} d(z), \quad z \in \mathrm{D}_{1 / 4}^{n} .
$$

Let $V_{\epsilon}$ be the set of points $z \in \mathrm{D}_{1 / 4}^{n}$ with $d(z)<\epsilon$. Let $\hat{V}_{\epsilon}$ be the set of $z \in \mathrm{D}^{n}$ with vertical distance $d_{v}(z)<\epsilon$. We have

$$
\operatorname{vol}\left(V_{\epsilon}\right) \leq \operatorname{vol}\left(\hat{V}_{c_{1} \epsilon}\right)=\pi\left(c_{1} \epsilon\right)^{2} \operatorname{vol}\left(\mathrm{D}^{n-1}\right)=\tilde{c} \epsilon^{2} .
$$

Let $\chi, 0 \leq \chi \leq 1$, be a smooth function such that $\chi(t)=1$ for $t>1$, $\chi(t)=0$ for $t<1 / 4$, and $\left|\chi^{\prime}\right|<2$. Let $\chi_{\epsilon}(z)=\chi\left(\frac{d(z)}{\epsilon}\right)$. Then $\mid \chi_{\epsilon}\left(z^{\prime}\right)-$ $\chi_{\epsilon}(z) \mid \leq \frac{2\left|z^{\prime}-z\right|}{\epsilon}$. Let $\chi_{\epsilon}^{*}$ be a regularization of $\chi_{\epsilon}$ such that $\chi_{\epsilon}^{*}$ vanishes on $V_{\epsilon / 8} \cap \mathrm{D}_{1 / 2}^{n}$ and $\chi_{\epsilon}^{*}=1$ on $\mathrm{D}_{1 / 2}^{n} \backslash V_{3 \epsilon / 2}$. Note that $0 \leq \chi_{\epsilon} \leq 1$ and $\left|\nabla \chi_{\epsilon}^{*}\right| \leq 2 / \epsilon$.

Let $\varphi$ be a smooth $(n, n-2)$-form supported in $\mathrm{D}_{1 / 4}^{n}$. Let $\frac{1}{p}+\frac{1}{q}=1$. Since $p \geq 2$ and $1 \leq q \leq 2$, we have

$$
\begin{aligned}
\left|\int_{\mathrm{D}^{n}} \chi_{\epsilon}^{*} A \wedge \bar{\partial} \varphi\right| & =\left|\int_{\mathrm{D}^{n}} A \wedge \bar{\partial} \chi_{\epsilon}^{*} \wedge \varphi\right| \\
& \leq c_{0}\|\varphi\|_{L^{\infty}}\left(\int_{V_{3 \epsilon / 2}}|A|^{p} d V\right)^{1 / p}\left(\int_{V_{\zeta_{\epsilon} / 2}}\left|\bar{\partial} \chi_{\epsilon}^{*}\right|^{q} d V\right)^{1 / q} \\
& \leq c_{1}\|\varphi\|_{L^{\infty}}\left(\int_{V_{3^{\prime} / 2}}|A|^{p} d V\right)^{1 / p} \epsilon^{\frac{2}{q}-1} \rightarrow 0, \quad \text { as } \epsilon \rightarrow 0 .
\end{aligned}
$$


Therefore, $B$, the trivial extension of $\frac{\bar{\partial} f}{f}$ by 0 on $f^{-1}(0)$, is $\bar{\partial}$-closed on $\mathrm{D}_{1 / 4}^{n}$ (and hence on $\left.\mathrm{D}^{n}\right)$.

We are ready to show that $r$ is holomorphic. By Proposition $\mathrm{C}$ and remarks 3(a)-(b), the uniform $L^{p}$ bound for $A_{L}$ in $\bar{\partial}\left(\left.f\right|_{L \cap \mathrm{D}^{n}}\right)=A_{L} f$, implies that $\frac{\partial f}{\partial \bar{z}_{j}}=B_{j} f$ on $\mathrm{D}^{n}$. Moreover, for all $z_{j}$-lines $L$ and the $\epsilon$-neighborhood $L_{\epsilon}$ of $L$ in $C^{n}$ with the sup-norm, $\left\|\left.B_{j}\right|_{L_{\epsilon} \cap D}\right\|_{L^{p}} \leq\left(\pi \epsilon^{2}\right)^{\frac{n-1}{p}} M$. By remark 3(d), all solutions $u$ to $\bar{\partial} u=B$, which exist, are in $C_{\mathrm{loc}}^{\alpha}\left(\mathrm{D}^{n}\right)$. Thus $e^{-u} f$ is holomorphic on $\mathrm{D}^{n} \backslash f^{-1}(0)$, and hence on $\mathrm{D}^{n}$ by Rado's theorem. Since the zero set of $e^{-u} f$ is given by the graph $z_{n}=r(' z)$, then $r$ is holomorphic.

Remarks. (a) The above estimates on two distances $d(z), d_{v}(z)$ are crucial. One can obtain $|f(z)| \leq c\left\|A_{L_{j}}\right\|_{L^{p}} d(z)^{1-\frac{2}{p}}$ from equations $\bar{\partial}\left(\left.f\right|_{L_{j}}\right)=A_{L_{j}} f$ by the well-known estimates stated at the beginning of Section 2 , without using an integrating factor. When $p \geq 3+\sqrt{5}$ (i.e $2\left(1-\frac{1}{p}\right)\left(1-\frac{2}{p}\right) \geq 1$ ) that estimate insures the above lemma, since as before

$$
\begin{aligned}
& \left|\int_{D^{n}} \chi_{\epsilon}^{*} A \wedge \bar{\partial} \varphi\right| \\
& \quad \leq c_{0}\|\varphi\|_{L^{\infty}}\left(\int_{V_{\zeta_{\epsilon} / 2}}|A|^{p} d V\right)^{1 / p}\left(\int_{V_{3 \epsilon / 2}}\left|\bar{\partial} \chi_{\epsilon}^{*}\right|^{q} d V\right)^{1 / q} \\
& \quad \leq c_{0}\|\varphi\|_{L^{\infty}}\left(\int_{V_{\zeta_{\epsilon} / 2}}|A|^{p} d V\right)^{1 / p}\left(\int_{\hat{V}}\left|\bar{\partial} \chi_{\epsilon}^{*}\right|^{q} d V\right)^{1 / q} \\
& \quad \leq c_{2}\|\varphi\|_{L^{\infty}}\left(\int_{V_{\zeta_{\epsilon} / 2}}|A|^{p} d V\right)^{1 / p} \epsilon^{2\left(1-\frac{1}{p}\right)\left(1-\frac{2}{p}\right)-1} \rightarrow 0, \quad \text { as } \epsilon \rightarrow 0 .
\end{aligned}
$$

(b) To obtain the $\bar{\partial}$-closedness of the trivial extension of $\frac{\bar{\partial} f}{f}$ on $\mathrm{D}^{n}$, it is crucial to assume that $\bar{\partial}\left(\left.f\right|_{L}\right)=A_{L} f$ on $L \cap \mathrm{D}^{n}$ with $\left\|A_{L}\right\|_{L^{p}} \leq M$ for all $z_{j}$-lines and for all $j=1, \ldots, n$. Take $n=2$ as an example. We do not know if the trivial extension is $\bar{\partial}$-closed if the $\bar{\partial}$-inequality is assumed on all $z_{1}$-lines and if the $\bar{\partial}$-inequality on all $z_{2}$-lines is replaced by $\frac{\bar{\partial} f}{f} \in L^{p}\left(\mathrm{D}^{2}\right)$ (for any $2<p<\infty)$. The latter insures $\bar{\partial}\left(\left.f\right|_{L}\right)=A_{L} f$ on $L \cap \mathrm{D}^{2}$ with $A_{L} \in L^{p}$ for almost all $z_{2}$-lines $L$, but the $L^{p}$ norms of $A_{L}$ might not be bounded as $L$ varies.

\subsection{End of proof of Theorem $A$}

The theorem is local. We may assume that $f$ is defined on $\mathbf{D}^{n}$. For each $z \in \mathbf{D}^{n}$, denote by $L_{j}(z)$ the $z_{j}$-line that is parallel to the $z_{j}$-axis and contains $z$. By 
one-dimensional result, we know that for each $p \in \mathrm{D}^{n}$ and each complex line $L=L_{j}(p)$, there is a continuous function $u$ so that $\left.e^{-u} f\right|_{L}$ is holomorphic on $L \cap \Omega$. Define the order of vanishing of $\left.f\right|_{L}$ at $p$ to be the vanishing order of $\left.e^{-u} f\right|_{L}$ at $p$, which is independent of the choice of $u$. Let $N_{p}=\infty$ if $\left.f\right|_{L_{j}(p)} \equiv 0$ for all $j$; otherwise let $N_{p}$ be the smallest integer such that for some $L=L_{j}(p),\left.f\right|_{L}$ vanishes to order $N_{p}$ at $p$.

Assume that $f(0)=0$. Since we consider only lines parallel to the coordinate axes it is possible that $N_{0}$ is infinite although $f$ is not identically 0 . By permuting the coordinates, we may assume that $z_{n}=0$ is a zero of $f\left(0, z_{n}\right)$ of order $N_{0}$.

(i) Case $N_{0}<+\infty$.

When $N_{0}=1$, near the origin, $f^{-1}(0)$ is a smooth complex hypersurface by Lemma 3.1(i) and Lemma 3.2.

Assume that $V=f^{-1}(0)$ is a complex variety near $p$ if $N_{p}<N$.

If $r_{1}=\cdots=r_{N}$ then by Lemma 3.1(ii), there is a continuous $N$-th root $f^{1 / N}(z)=\left(z_{n}-r(' z)\right) v(z)$, which still satisfies the $\bar{\partial}$-differential inequality. We have $N_{0}\left(f^{1 / N}\right)=N_{0}(f) / N=1$. Hence $f^{-1}(0)$ is a smooth complex hypersurface near the origin.

Suppose $N_{0}=N \geq 2$. Without loss of generality, we may assume by Lemma 3.1(i) that the zero set of $f$ in $\mathrm{D}^{n}$ is given by $z_{n}=r_{j}\left({ }^{\prime} z\right), j=1, \ldots, N$, counting multiplicity.

We already proved the assertion when $r_{1}=\cdots=r_{N}$. So we may assume that not all of $r_{1}(a), \ldots, r_{N}(a)$ are the same for some $a$. Let $k$ be the largest integer such that $r_{1}(' z), \ldots, r_{N}(' z)$ have $k$ distinct elements for some ' $z$. Define

$$
h(' z)=\Pi_{1 \leq \alpha \neq \beta \leq k}\left(r_{j_{\alpha}}(' z)-r_{j_{\beta}}(z)\right),
$$

when $r_{j_{1}}(' z), \ldots, r_{j_{k}}(' z)$ are distinct. Rename the distinct $k$ elements by $r_{1}^{*}(' z)$, $\ldots, r_{k}^{*}(' z)$. For clarity, we do not define $r_{1}^{*}\left({ }^{\prime} z\right), \ldots, r_{k}^{*}\left({ }^{\prime} z\right)$ when $\left\{r_{1}(' z), \ldots\right.$, $\left.r_{N}(z)\right\}$ has less than $k$ distinct elements, in which case we set $h(' z)=0$.

We first want to show that $h$ is holomorphic away from $h^{-1}(0)$. Assume that $h(a) \neq 0$. Since $k \geq 2$ then $N_{\left(a, r_{j}^{*}(a)\right)}<N$. Thus $f^{-1}(0)$ is a complex variety near each $\left(a, r_{j}^{*}(a)\right)$. By Lemma 3.1(i), for' $z$ close to $a, f\left(' z, z_{n}\right)$ has at least one zero near each $r_{j}^{*}(a)$. Therefore, by the definition of $k, f\left(' z, z_{n}\right)$ has exactly one zero near each $r_{j}^{*}(a)$ for $z$ sufficiently close to $a$. Thus near each $\left(a, r_{j}^{*}(a)\right)$ the complex variety $f^{-1}(0)$ must be smooth, and near $a$ we redefine $r_{j}^{*}\left(z^{\prime}\right)$ such that they become holomorphic in $z^{\prime}$. In particular $h$ is holomorphic away from its zero set.

Next we want to show that $h$ is continuous. Fix $a \in \mathrm{D}^{n-1}$ with $h(a)=0$. Given a sequence $a^{u}$ approaching to $a$ as $u \rightarrow \infty$ we want to show that $\lim _{u \rightarrow \infty} h\left(a^{u}\right)=0$. Without loss of generality we may assume that $h\left(a^{u}\right) \neq 0$ 
for all $u$. Let $b_{1}, \ldots, b_{d}$ be distinct zeros of $f\left(a, z_{n}\right)$ in $\mathrm{D} \ni z_{n}$. Let $m_{j}$ be the multiplicity of the zero $z_{n}=b_{j}$ of $f\left(a, z_{n}\right)$. Put $s=\frac{1}{4} \min \left\{\mid b_{j}-\right.$ $\left.b_{k}|, 1-| b_{k} \mid: 1 \leq k \neq j \leq d\right\}$. By Lemma 3.1(i), we can choose $t>0$ so that the zero set of $f$ in $\left(a+\mathrm{D}_{t}^{n-1}\right) \times\left(b_{j}+\mathrm{D}_{s}\right)$ is given by $z_{n}=w_{j \alpha}\left({ }^{\prime} z\right)$, $1 \leq \alpha \leq m_{j}$; moreover $\lim _{z \rightarrow a} w_{j \alpha}(z)=b_{j}$. Therefore the limit of any convergent subsequence $\left(r_{1}^{*}\left(a^{u_{j}}\right), \ldots, r_{k}^{*}\left(a^{u_{j}}\right)\right), j=1,2, \ldots$ must have the form $\left(b_{l_{1}}, \ldots, b_{l_{k}}\right)$. Since $\left\{b_{l_{1}}, \ldots, b_{l_{k}}\right\} \subset\left\{b_{1}, \ldots, b_{d}\right\}$ and $d<k$, we conclude that $\lim _{j \rightarrow \infty} h\left(a^{u_{j}}\right)=\prod_{1 \leq \alpha \neq \beta \leq k}\left(b_{l_{\alpha}}-b_{l_{\beta}}\right)=0$. Therefore $h$ is continuous and hence holomorphic by Rado's theorem.

Away from the zero set of $h$, the symmetric polynomials of $r_{1}^{*}(' z), \ldots, r_{k}^{*}\left({ }^{\prime} z\right)$ are holomorphic in' $z$ (in fact we proved that away from the zero set, $r_{1}^{*}(' z), \ldots$, $r_{k}^{*}(z)$ can be locally rearranged to become holomorphic). Since the symmetric polynomials are bounded, they extend holomorphically to $\mathrm{D}^{n-1}$, by the removable singularity theorem. By the removable singularity theorem again, $P(z)=\left(z_{n}-r_{1}^{*}(' z)\right) \ldots\left(z_{n}-r_{k}^{*}(' z)\right)$, defined for $h(' z) \neq 0$ only, extends holomorphically to $\mathrm{D}^{n}$. Now we want to find a neighborhood $U$ of the origin such that $P^{-1}(0) \cap U=f^{-1}(0) \cap U$. By definition $P^{-1}(0) \cap \mathrm{D}^{n} \backslash h^{-1}(0)=$ $f^{-1}(0) \cap \mathrm{D}^{n} \backslash h^{-1}(0)$. Since $h \not \equiv 0$ the closure of $f^{-1}(0) \backslash h^{-1}(0)$ in $\mathrm{D}^{n}$ is $f^{-1}(0) \cap \mathrm{D}^{n}$ by Lemma 3.1(i). Since $P\left(0, z_{n}\right) \not \equiv 0$ there is a polydisc $U=$ $\mathrm{D}_{\delta}^{n-1} \times \mathrm{D}_{\epsilon}$ such that $P^{-1}(0) \cap U$ is given by a branched-covering over $\mathrm{D}_{\delta}^{n-1}$. So $\overline{\left(P^{-1}(0) \backslash h^{-1}(0)\right)} \cap U=P^{-1}(0) \cap U$. Therefore $f^{-1}(0) \cap U=P^{-1}(0) \cap U$, which is a complex variety.

(ii) Integrating factor and case $N_{0}=+\infty$.

We now go back to the main question which is the $\bar{\partial}$-closedness of $B$, (which was already central in Lemma 3.2).

We shall use the following result of Demailly ([2], Lemma 6.9), whose proof is similar to the argument in Lemma 3.2, with an induction on the dimension of the analytic set. Let $\Omega$ be an open set in $C^{n}$, and let $E$ be an analytic subset of $\Omega$ of dimension $<n$. Let $g$ be a $(0,1)$-form defined on $\Omega$ with $L^{2}$ coefficients. If $g$ is $\bar{\partial}$-closed on $\Omega \backslash E$ then $g$ is $\bar{\partial}$-closed on $\Omega$.

We now assume that Theorem $\mathrm{A}$ is proved in dimension $n-1$ and we want to establish it in dimension $n$. Assume that there exists $a=\left(' a, a_{n}\right) \in \mathrm{D}^{n}$ with $f(a) \neq 0$. Let $X=\left\{z \in D^{n-1}: f\left(' z, a_{n}\right)=0\right\}$. By the induction hypothesis, this is a proper analytic subset of $\mathrm{D}^{n-1}$. If $p=\left({ }^{\prime} p, p_{n}\right)$ is such that $N(p)=+\infty$ then $f\left({ }^{\prime} p, \cdot\right) \equiv 0$, so' $p \in X$.

Set $Z=X \times \mathrm{D}$. This is a proper analytic subset of $\mathrm{D}^{n}$, and if $p \notin Z$ then $N(p)<+\infty$.

Let $B$ be the trivial extension of $\frac{\bar{\partial} f}{f}$, as considered earlier. We already know that $B \in L_{(0,1)}^{p}\left(\mathrm{D}^{n}\right)$. We claim that $B$ is $\bar{\partial}$-closed on $\mathrm{D}^{n}$. Applying Demailly's result to $\Omega=\mathrm{D}^{n} \backslash Z$, it follows from the analyticity of $f^{-1}(0)$ (at points where 
$N$ is finite) shown in (i) that $B$ is $\bar{\partial}$-closed on $\mathrm{D}^{n} \backslash Z$. Applying Demailly's result again, it follows that $B$ is $\bar{\partial}$-closed on $\mathrm{D}^{n}$, as desired. As at the end of the proof of Lemma 3.2, for some locally defined $u \in C_{\mathrm{loc}}^{\alpha}, e^{-u} f$ is holomorphic.

The proof of Theorem A is complete.

\subsection{Vector-valued $f$ defined on $\mathrm{C}^{n}$ when $f^{-1}(0)$ is real analytic}

We turn to the case that $f$ is vector-valued and defined on a domain $\Omega \subset \mathrm{C}^{n}$ with $n>1$. Here our result is far from complete. We will treat only the case that $f^{-1}(0)$ is already real analytic. To show the complex analyticity, we will impose condition on $\bar{\partial}\left(f_{\gamma}\right)$ for all germs of complex curve, not just lines. But we will not need the uniform bound on $L^{p}$-norms.

First we recall some basic results on real analytic sets, which can be found in [8].

Let $V_{0}$ be an irreducible germ of real analytic set at $0 \in \mathrm{R}^{m}$ of dimension $k$. (We will take $\mathrm{R}^{m}=\mathrm{R}^{2 n} \subset \mathrm{C}^{2 n}$ soon.) Then there is an open subset $D$ of $\mathrm{R}^{m}$ and a closed real analytic set $V$ in $D$ which represents the germ, with $\operatorname{dim} V_{x} \leq k$ for all $x \in V$. Denote by $V^{*}$ the set of points in $V$ at which $V$ is a smooth submanifold of dimension $k$. We also need the complexification $\tilde{V}_{0}$ of the germ $V_{0}$ : there exists a unique irreducible germ $\tilde{V}_{0}$ of complex variety in $\mathrm{C}^{m}$ with $\tilde{V}_{0} \cap \mathrm{R}^{m}=V_{0}$ ([8], Proposition 1, p. 91). In particular, one can choose an open subset $\tilde{D}$ of $\mathrm{C}^{m}$ such that $\tilde{V}_{0}$ is represented by an irreducible closed complex variety $\tilde{V} \subset \tilde{D}$ of pure dimension $k$.

We need the following result which is essentially in [3].

Lemma 3.3. Let $V_{0}$ be an irreducible germ of real analytic variety at $0 \in \mathrm{C}^{n}$ of dimension $k$, represented by a closed real analytic set $V$ in $U \ni 0$ of the same dimension. If $V^{*}$ is a complex submanifold of $\mathrm{C}^{n}$, then $V$ is a complex variety at 0 .

Proof. It suffices to find a germ of complex variety $\hat{V}$ at 0 that is contained in $V$ and has the same real dimension as $V$. Then the irreducibility of $V$ implies that two germs must agree ([8], Proposition 7, p. 41). We will adapt an argument in [3] to construct $\hat{V}$.

Choose a polydisc $D \subset U$, centered at 0 , such that $V \cap D$ is the zero set of a real function $r(z, \bar{z})$ which is a convergent power series on $D \times D$. Thus $Q_{w} \stackrel{\text { def }}{=}\{z \in D: r(z, \bar{w})=0\}$ is a complex variety in $D$ for each $w \in D$. Choose a domain $\tilde{D}_{1} \subset \mathrm{C}^{2 n}$ and an irreducible complex variety $\tilde{V}$ in $\tilde{D}_{1}$ such that $\tilde{V}_{0}$ is the complexification of $V_{0}$. We may assume that $\tilde{D}_{1} \cap \mathrm{R}^{2 n} \subset D$ and $\tilde{V} \cap \mathrm{R}^{2 n}=V \cap \tilde{D}_{1}$.

We want to show that $Q_{w} \supset V^{*} \cap \tilde{D}_{1}$ for $w \in V^{*} \cap \tilde{D}_{1}$. To that end, choose a biholomorphic map $\varphi: \mathrm{D}^{k} \rightarrow W \subset V^{*}$ with $\varphi(0)=w$. Then $r(\varphi(t), \overline{\varphi(t)})=$ 0 for $t \in \mathrm{D}^{k}$. Thus $r(\varphi(t), \overline{\varphi(0)})=0$, i.e. $r(\cdot, w)$ vanishes on some open 
subset $W$ of $V^{*}$. Note that $W \subset \mathrm{C}^{n}=\mathrm{R}^{2 n}+i 0$ is embedded in $\mathrm{C}^{2 n}$. Thus $W$ is a totally real submanifold in $\tilde{V}$ of dimension $k$. As a function in $(x, y) \in$ $\tilde{V}^{*}, r(x+i y, w)$ vanishes on an open subset of $\tilde{V}^{*}$. Since $\tilde{V}^{*}$ is connected, then $r(x+i y, w)$ vanishes on $\tilde{V}$, which implies that $r(z, w)$ vanishes for $z \in V^{*} \cap \tilde{D}_{1}\left(\subset \tilde{V} \cap \mathrm{R}^{2 n}\right)$. Now we go back to $C^{n}$

We just proved that $S \stackrel{\text { def }}{=} \cap_{w \in V^{*} \cap \tilde{D}_{1}} Q_{w} \supset V^{*} \cap \tilde{D}_{1}$. For $w \in V^{*} \cap \tilde{D}_{1}$ we have $S \subset Q_{w}$, i.e. $z \in Q_{w}\left(\Leftrightarrow w \in Q_{z}\right)$ for $z \in S$. Thus $V^{*} \cap \tilde{D}_{1} \subset Q_{z}$ for $z \in S$. Now

$$
V^{*} \cap \tilde{D}_{1} \subset \cap_{w \in S} Q_{w} \stackrel{\text { def }}{=} \hat{V} .
$$

Fix $z \in \hat{V} \subset S$. We have $z \in S$ and get $z \in(\hat{V}=) \cap_{w \in S} Q_{w} \subset Q_{z}$. Now $z \in Q_{z}$ implies that $r(z, \bar{z})=0$, i.e $z \in V$. Finally, $V^{*} \cap \tilde{D}_{1} \subset \hat{V} \subset V$. Since $V^{*}$ has pure complex dimension $k$ and $0 \in \bar{V}^{*}$ then $\hat{V}$ has real dimension at least $2 k$, which is already the dimension of $V$. Since $V$ is irreducible then $V=\hat{V}$ as germs at 0 .

As a consequence of Lemma 2.1 and Lemma 3.3, we have

Proposition 3.4. Let $2<p \leq \infty$. Let $f=\left(f_{1}, f_{2}, \ldots, f_{m}\right)$, where $f_{j}$ are continuous functions on a domain $\Omega \subset \mathrm{C}^{n}$, and let $\Omega^{*}=\Omega \backslash f^{-1}(0)$. Assume that for each smooth holomorphic curve $\gamma$ in $\Omega$ (not necessarily closed in $\Omega$ ), $\bar{\partial}\left(\left.f\right|_{\gamma}\right)=f A_{\gamma}$ holds in the distribution sense on $\gamma \cap \Omega^{*}$, where $A_{\gamma}$ is an $m \times m$ matrix of $(0,1)$-forms whose coefficients are in $L_{\mathrm{loc}}^{p}(\gamma)$. If $f^{-1}(0)$ is a real analytic variety in $\Omega$, then it is a complex variety.

Proof. Assume that $f(0)=0$. We want to show that $f^{-1}(0)$ is a complex variety near the origin.

Decompose the germ of $f^{-1}(0)$ at 0 as $V_{0}^{1} \cup \cdots \cup V_{0}^{k}$, where $V_{0}^{j}$ are germs of irreducible real analytic sets at 0 and $V_{0}^{j}$ is not contained in $\cup_{k \neq j} V_{0}^{k}$. (For real analytic sets, the irreducible decomposition may exist at the germ level only!) Choose an open neighborhood $D$ of 0 and a closed real analytic set $V_{j}$ in $D$ such that $V_{0}^{j}$ is the germ of $V_{j}$ at 0 . Since $f^{-1}(0)$ and $\cup V_{j}$ represent the same germ at 0 there exists an open set $D \ni 0$ such that $V \stackrel{\text { def }}{=} f^{-1}(0) \cap D=\cup V_{j} \cap D$. Let $p_{j}$ be the dimension of $V_{0}^{j}$. We may assume that $V_{j}$ has dimension $p_{j}$ too. Since $\operatorname{dim} \cup_{k \neq j} V_{0}^{k} \cap V_{0}^{j}<\operatorname{dim} V_{0}^{j}$, we may choose $D$ so small that $\operatorname{dim} \cup_{k \neq j} V_{k} \cap V_{j}<\operatorname{dim} V_{j}$. Let $V_{j}^{*}$ be the set of points in $V_{j}$ near which $V_{j}$ is a real submanifold of dimension $p_{j}$. Put $\tilde{V}_{j}=V_{j}^{*} \backslash \cup_{k \neq j} V_{k}$. Each tangent vector in $T_{x} \tilde{V}_{j}$ is the tangent vector of some real analytic curve $\gamma$ in $\tilde{V}_{j}$. Let $\tilde{\gamma}$ be the complexification of $\gamma$. By the one-dimensional result, $f^{-1}(0) \cap \tilde{\gamma}$ is a complex variety in $\tilde{\gamma}$. Since $f^{-1}(0) \cap \tilde{\gamma}$ is not isolated, then $\tilde{\gamma}$ is contained in $f^{-1}(0)$ (one may assume that $\tilde{\gamma}$ is connected). Therefore, $T_{x} \tilde{V}_{j}$ is complex linear subspace 
of $T_{x} \mathrm{C}^{n}$. Since $\tilde{V}_{j}$ is dense in $V_{j}^{*}$, then $V_{j}^{*}$ is a complex submanifold of $\mathrm{C}^{n}$. By the previous lemma, we know that each $V_{j}$ is a complex variety.

\section{Remarks on some uniqueness or finite type results for $J$-holomorphic curves}

In the theory of $J$-holomorphic curves inequalities of the type

$$
|\bar{\partial} f| \leq c(z)|f|
$$

occur, as well as inequality of the type

$$
|\bar{\partial} f| \leq c(f)|\partial f| .
$$

The more general inequality $|\bar{\partial} f| \leq c(z, f)|\partial f|$ can, of course, be reduced to (T2) by considering $(z, f(z))$ instead of $f(z)$.

If one has an $L^{p}$-bound for $\partial f$ and an estimate $|c(f)| \leq|f|$, reversing roles, one can reduce (T2) to (T1).

An example is the following: Assume that $v_{1}: \mathrm{D} \rightarrow\left(\mathrm{R}^{2 n}, J\right)$ is an embedded $J$-holomorphic curve. After changing variables we assume that $v_{1}(z)=$ $(z, 0, \ldots, 0) \in \mathrm{C}^{n} \simeq \mathrm{R}^{2 n}$, and that $J(z, 0, \ldots, 0)=J_{s t}$ (the standard complex structure on $\left.\mathrm{C}^{n}\right)$. Let $v_{2}: \mathrm{D} \rightarrow\left(\mathrm{R}^{2 n}, J\right)$ be continuous. Let $E$ be a closed subset of $\mathrm{D}$ with $0 \in E$. Assume that on $E v_{2}(z)=v_{1}(z)=(z, 0, \ldots, 0)$ and that $v_{2}$ is $J$-holomorphic on $\mathrm{D} \backslash E$ (but make no a-priori assumption of $J$-holomorphicity of $v_{2}$ at the boundary points of $E$ ). It follows from Lemma 2.1 in Part One that if $\nabla v_{2} \in L^{p}$ for some $p>2$ then near $0, v_{2} \equiv v_{1}$ or $v_{2}-v_{1}$ vanishes to finite order only. Indeed, the equation for $J$-holomorphy is $\frac{\partial v}{\partial \bar{z}}=Q(v) \frac{\overline{\partial v}}{\partial z}$, and $Q(z, 0, \ldots, 0) \equiv 0$, hence $Q\left(v_{1}\right) \equiv 0$ (with $Q \in M_{n, n}(\mathbf{C})$ and of the smoothness of $J$ ). So

$$
\begin{aligned}
\left|\frac{\partial\left(v_{2}-v_{1}\right)}{\partial \bar{z}}\right| & =\left|\frac{\partial v_{2}}{\partial \bar{z}}\right|=\left|Q\left(v_{2}\right) \frac{\overline{\partial v_{2}}}{\partial z}\right|=\left|\frac{\partial v_{2}}{\partial z}\right|\left|Q\left(v_{2}\right)-Q\left(v_{1}\right)\right| \\
& \leq c(z)\left|v_{2}-v_{1}\right|, \quad \text { with } \quad c \in L^{p} .
\end{aligned}
$$

However the above result (that $v_{2} \equiv v_{1}$ or $v_{2}-v_{1}$ vanishes to finite order only) follows immediately from the Corollary to Theorem C of Part Two (applied to $\left(z, v_{j}\right)$ rather than $\left.v_{j}(z)\right)$. Indeed this Corollary implies that in fact $v_{2}$ is $J$-holomorphic (i.e. the exceptional set $E$ can be removed). Then, the result is well-known. 


\section{Part 2. Removable singularities for $J$-holomorphic maps and Rado's Theorem}

\section{Introduction}

Let $\Omega$ be an open set in C. A subset $E \subset \Omega$ is said to be a polar set if for any $z \in \Omega$ there exists a subharmonic function $\lambda$ defined on a neighborhood $V$ of $z$ (not identical to $-\infty$ near $z$ ) such that $E \cap V \subset \lambda^{-1}(-\infty)$. If $E$ is closed in $V$ one can take $\lambda$ to be continuous as a map from $V$ into $\{-\infty\} \cup \mathrm{R}$ (and $\left.E \cap V=\lambda^{-1}(-\infty)\right)$.

If $E$ is a polar subset of $\Omega$, then there exists a subharmonic function $\mu$ on $\Omega \mu \not \equiv-\infty$ such that $E \subset \mu^{-1}(-\infty)$. Indeed local polarity implies global polarity, and this is an easy result unlike Josefson's theorem on pluripolarity. (Hint: Take a locally finite covering of $\Omega$ and $\lambda_{j}$ corresponding subharmonic functions and solve $\Delta \lambda=\sum_{j} \Delta \lambda_{j}$.)

In Part Two all almost complex structures are of class $C^{1}$, and all $J$ holomorphic curves $u$ are of class $C^{1}$ at least. So $u$ are of class $C^{1, \alpha}$ for any $\alpha<1$ (See [6]).

THEOREM B. Let $\Omega$ be an open subset in $\mathrm{C}$ and let $\mathrm{E}$ be a closed polar subset of $\Omega$. Let $u$ be a continuous map from $\Omega$ into an almost complex manifold $(M, J)$ with $J$ of class $C^{1}$. If $u$ is $J$-holomorphic on $\Omega \backslash E$ then it is $J$ holomorphic on $\Omega$.

The next theorem is also about removable singularities but this time in terms of the target space. It is a theorem in the style of the classical theorem of Rado that states that a continuous function that is holomorphic off its zero set is holomorphic.

Definitions. 1) A closed subset $\mathscr{C}$ of an almost complex manifold $(M, J)$ will be said to be a Rado subset of $(M, J)$ if and only if the following holds: If $u$ is a continuous map from a connected open subset $\Omega$ of $C$ into $M$ such that $u$ is $J$-holomorphic at any point $z$ such that $u(z) \notin \mathscr{C}$ then $u$ is $J$-holomorphic on $\Omega$ or $u(\Omega) \subset \mathscr{C}$.

2) A subset $L$ of $(M, J)$ is said to be locally exact $J$-pluripolar, if and only if for any $q \in L$ there exits a neighborhood $V$ of $q$ and a $J$-holomorphic function $\rho$ defined on $V$, continuous as a map from $V$ into $\{-\infty\} \cup \mathrm{R}$, such that $\rho \not \equiv-\infty$ near $q$ and $L \cap V=\rho^{-1}(-\infty)$. (The notion of $J$-plurisubharmonicity will be recalled later.)

THeOREM C. Let $\mathscr{C}$ be a closed subset of an almost complex manifold $(M, J)$. Assume that there is a discrete subset $S$ of $\mathscr{C}$ such that $\mathscr{C} \backslash S$ is locally exact $J$-pluripolar then $\mathscr{C}$ is a Rado subset. 
Corollary. Let $(M, J)$ be an almost complex manifold of class $C^{1}$. The proper image of an open subset of $C$ under a J-holomorphic map of class $C^{2}$ is a Rado subset. Discrete subsets of $M$ are Rado subsets.

By the proper image of an open subset $\Omega$ of $C$ under a $J$-holomorphic map $u$, we mean that the map $u: \Omega \rightarrow M$ is proper.

Proposition B, which asserts the existence of a small neighborhood $\omega \subset D$ of the origin such that either the $v$ is $J$-holomorphic on $\omega$ or $v(\omega)$ is contained in $u$ (D), follows from the above Corollary. In general, one cannot take $\omega$ to be D.

Two results on $J$-pluripolarity will be used. The first one will allow us to prove that Theorem $\mathrm{C}$ follows from Theorem $\mathrm{B}$. The second one shows that the corollary follows immediately from Theorem $\mathrm{C}$.

a) If $p \in(M, J)$ there exists a $J$-plurisubharmonic function $\rho$ defined on a neighborhood of $p$ and continuous away from $p$ such that $\rho^{-1}(-\infty)=\{p\}$. This is a local question. We can assume that $M=\mathrm{R}^{2 n} \simeq \mathrm{C}^{n}, p=0$, and $J(0)=J_{s t}$. Then for $A>0$ sufficiently large one can take $\rho(Z)=\log |Z|+$ $A|Z|$ (Chirka, see a proof in [6] Lemma 1.4, p. 2401).

b) If $\Sigma$ is a (germ of) embedded $J$-holomorphic $C^{2}$ disc and $q \in \Sigma$ there exists a $J$-plurisubharmonic function $\rho$ defined in a neighborhood $V$ of $q$, which is continuous map from $V$ into $\{-\infty\} \cup \mathrm{R}$, such that $\Sigma \cap V=\rho^{-1}(-\infty)$ ([10]).

Recall that a function $\lambda$ defined on an open set of $(M, J)$ is said to be $J$-plurisubharmonic if and only if $\lambda$ is upper semicontinuous and its restriction to any $J$-holomorphic curve is subharmonic (i.e if $u: \mathrm{D} \rightarrow(M, J)$ is $J$ holomorphic then $\lambda \circ u$ is subharmonic). For a smooth function $\lambda$ the condition is that for any tangent vector $T$ of $M$ at a point $q \in M:\left(d d_{J}^{c} \lambda\right)_{q}(T, J(q) T) \geq$ 0. See Corollary 1.1 in [6].

We shall repeatedly use the crucial formula: If $u$ is a $C^{1} J$-holomorphic map and $\lambda$ is a $C^{2}$ function then

$$
\Delta(\lambda \circ u)(z)=\left[d d_{J}^{c} \lambda\right]_{u(z)}\left(\frac{\partial u}{\partial x}(z), J(u(z)) \frac{\partial u}{\partial x}(z)\right)
$$

(formula (1.2) in [6], p. 2399), where $d_{J}^{c} \lambda(Y)=-d \lambda(J Y)$. In [6] there is no claim of originality for the above formula, and the reference is given for the convenience of the reader.

REMARKS. 1) We do not know whether $J$-holomorphic curves with cusps are (locally) $J$-pluripolar. It would simplify our proof of Theorem C.

2) Theorem B is a generalization of the proof of removal of isolated singularities for $J$-holomorphic maps that extend continuously. Its proof borrows 
from the known proof of the removal of isolated singularities, although some of arguments are slightly different from the usual ones even in that case.

\section{Proof of Theorem B}

\subsection{Preliminaries}

There is no claim of originality for the following lemmas. The first one is just the case for the classical Rado theorem, and the second one is a version of Rado's theorem. The third one is certainly very classical.

Lemma 2.1. Let $\Omega$ be an open set in $C$ and let $E$ be a closed polar subset of $\Omega$. If $v$ is a continuous function on $\Omega$ that is subharmonic on $\Omega \backslash E$, then $v$ is subharmonic on $\Omega$.

Proof. Let $\rho$ be a subharmonic function on $\Omega$ that is $-\infty$ exactly on $E$ and not identical $-\infty$ on any open set. Then $v=\left[\lim _{\epsilon \rightarrow 0^{+}}(v+\epsilon \rho)\right]^{*}$, where $*$ denotes upper semicontinuous regularization.

Lemma 2.2. Let $\Omega$ and $E$ be as in Lemma 2.1. If $u$ is a bounded harmonic function defined on $\Omega \backslash E$ then u extends to a harmonic function on $\Omega$.

Proof. Set $u^{*}(z)=\lim \sup _{\zeta \rightarrow z} u(\zeta)$ for $\zeta \in \Omega$. Then $u^{*}$ is subharmonic since $u^{*}=\left[\lim _{\epsilon \rightarrow 0^{+}}(u+\epsilon \rho)\right]^{*}$. Also $(-u)^{*}$ defined similarly is subharmonic. At any $z \in \Omega \backslash E, u^{*}(z)=u(z)$ and $(-u)^{*}(z)=-u(z)$. So by the mean value property for $u^{*}$ and $(-u)^{*}, u$ is obtained on any disc relatively compact in $\Omega$ by integration of $u$ against the Poisson kernel on its boundary (whose intersection with $\rho^{-1}(-\infty)$ has zero measure).

Lemma 2.3. Let $\Omega$ be a domain in $\mathrm{C}$ and let $E$ be a closed polar subset of $\Omega$. Let $g$ be a bounded subharmonic function on $\Omega$. If $\mu=\Delta g$ (it is a positive measure), then $\mu(E)=0$.

Proof. The question is local, so we may assume that $\Omega=\mathrm{D}$ and $\mu$ is a finite measure with compact support in $\overline{\mathrm{D}}$. Then $g=\mu * N+h$, where $N=\frac{\log |z|}{2 \pi}$ is the Newtonian potential and $h$ is harmonic on D. Let $\mu=\mu_{E}+\mu_{E^{c}}$ where $\mu_{E}$ is the restriction of the measure $\mu$ on $E$. We have to show that $\mu_{E}=0$. Since $g$ is bounded $\mu * N$ must be locally bounded on D. Since both $\mu_{E}$ and $\mu_{E^{c}}$ are positive (and only large positive values can occur to $-\log |z|$ ) both $\mu_{E} * N$ and $\mu_{E^{c}} * N$ (a priori in $L^{1}(\mathrm{D})$ ) must be locally bounded. But $\mu_{E} * N$ is harmonic off $E$. So by Lemma 2.2, $\mu_{E} * N$ extends to a harmonic function and so $\mu_{E}=\Delta\left(\mu_{E} * N\right)=0$. 


\subsection{The proof of Theorem $B$}

The question is local. We can assume that $M=\mathrm{R}^{2 n}, 0 \in \Omega, u(0)=0 \in \mathrm{R}^{2 n}$ and in $\mathrm{R}^{2 n} J(0)=J_{s t}$. We want to prove $J$-holomorphicity of $u$ at 0 .

Step 1. $\nabla u \in L_{\text {loc }}^{1}(\Omega)$. Here $\nabla u$ means the distributional gradient of $u$ on $\Omega$, not only its restriction to $\Omega \backslash E$. We identify $\mathrm{R}^{2 n}$ with coordinates $\left(x_{1}, y_{1}, \ldots, x_{n}, y_{n}\right)$ with $C^{n}$ via $z_{j}=x_{j}+i y_{j}$, and put $|Z|^{2}=\sum_{j=1}^{n}\left|z_{j}\right|^{2}$. Note that $|Z|^{2}$ is strictly $J$-plurisubharmonic near 0 (see [6], Lemma 1.3, p. 2400). Let $\varphi$ be either the function $\operatorname{Re} z_{j}$ or $\operatorname{Im} z_{j}$ for some $j \in\{1, \ldots, n\}$. Then $|Z|^{2}$ is $J$-plurisubharmonic near 0 and so is $\varphi+K|Z|^{2}$ if $K>0$ is sufficiently large.

In a neighborhood of $0 \in \mathrm{C},|Z|^{2} \circ u$ and $\left(\varphi+K|Z|^{2}\right) \circ u$ are subharmonic by Lemma 2.1 since off $E u$ is $J$-holomorphic. Therefore $\Delta\left(\left(\varphi+K|Z|^{2}\right) \circ u\right)$ and $\Delta\left(|Z|^{2} \circ u\right)$ both are positive measures, and $d \mu=\Delta(\varphi \circ u)$ is a (locally finite) measure. Hence $\nabla(\varphi \circ u) \in L_{\operatorname{loc}}^{1}(\Omega)$, since $\varphi \circ u$ is equal to $\left.\Delta(\varphi \circ u)\right|_{\mathrm{D}} * \frac{\log |z|}{2 \pi}$ modulo smooth functions and the distributional gradient $\nabla\left(d \mu * \frac{\log |z|}{2 \pi}\right)=$ $\left(\nabla \frac{\log |z|}{2 \pi}\right) * d \mu \in L_{\mathrm{loc}}^{1}(\Omega)$.

The last assertion is true for each coordinate function $\varphi$, so $\nabla u \in L_{\mathrm{loc}}^{1}(\Omega)$ as claimed.

Step 2. Now that we know that $\nabla(\varphi \circ u) \in L_{\text {loc }}^{1}$ we can show that $\nabla u \in$ $L_{\text {loc }}^{2}(\Omega)$.

Off $E$ (which has Lebesgue measure 0 since it is polar),

$$
\Delta(\lambda \circ u)(z)=\left[d d_{J}^{c} \lambda\right]_{u(z)}\left(\frac{\partial u}{\partial x}(z), J(u(z)) \frac{\partial u}{\partial x}(z)\right) .
$$

For $p$ in a neighborhood of 0 we have for any tangent vector $T$ at $p$

$$
d d_{J}^{c}|Z|^{2}(T, J(p) T) \geq C|T|^{2} .
$$

So $\left|\frac{\partial u}{\partial x}\right|^{2} \leq \frac{1}{C} \Delta\left(|Z|^{2} \circ u\right)$. Since $\frac{\partial u}{\partial y}=J \frac{\partial u}{\partial x}$ then $|\nabla u|^{2}$ is locally integrable in $\Omega$, i.e $\nabla u \in L_{\text {loc }}^{2}(\Omega)$.

Note: In step 2, global $L^{2}$ estimates are obtained on the complement of $E$. Step 1 is used to make sure that, roughly speaking, $E$ carries no part of the distributional gradient of $u$. This would be clear (by abrupt cutoff and differentiation) if one knew that $E$ has a basis of neighborhoods whose lengths of the boundaries tend to 0 . In that case step 1 is not needed. At any rate, our approach avoids using any non immediate property of polar sets.

Step 3. We now want to show that for any $r>0$ (we shall need to use $r>4) \nabla u \in L_{\text {loc }}^{r}(\Omega)$. Replacing $u$ by $u(\eta z)$ for $\eta$ small we can assume that $u$ 
is defined on the unit disc $\mathrm{D}$ and the condition for $J$-holomorphicity will give a differential inequality

$$
\left|\frac{\partial u}{\partial \bar{z}}\right| \leq c_{0}\left|\frac{\partial u}{\partial z}\right|
$$

where $c_{0}$ is small enough as will be said later. No more will be used.

Fix $\chi$ a cutoff function with $\chi \equiv 1$ near 0 in $C$ and with compact support in the unit disc. Let $S$ be the convolution with the singular kernel $\frac{-1}{\pi z^{2}}$. Then we know that

$$
\frac{\partial}{\partial z}(\chi u)=\frac{-1}{\pi z^{2}} *\left[\frac{\partial}{\partial \bar{z}}(\chi u)\right]=S\left(\frac{\partial}{\partial \bar{z}}(\chi u)\right)
$$

since $\chi u$ is in $L^{2}$ and of compact support. By the Calderon-Zygmund theory for $1<r<\infty$ and $h \in L^{r}$ (D)

$$
\|S(h)\|_{L^{r}(\mathrm{D})}=\left\|h *\left(\frac{-1}{\pi z^{2}}\right)\right\|_{L^{r}(\mathrm{D})} \leq \epsilon_{r}\|h\|_{L^{r}(\mathrm{D})} .
$$

For $r=2, \epsilon_{2}=1$. Write the differential inequality (A) as

$$
\frac{\partial u}{\partial \bar{z}}=-\alpha(z) \frac{\partial u}{\partial z}, \quad \text { with } \quad \alpha \in L^{\infty},|\alpha| \leq c_{0} .
$$

Although $\alpha$ depends on $u$ whose regularity we want to show, consider it to be a given function. After cutoff

$$
\frac{\partial \chi u}{\partial \bar{z}}+\alpha(z) \frac{\partial \chi u}{\partial z}=g
$$

where $g$ is a bounded function. So

$$
[\mathbf{1}+\alpha S] \frac{\partial \chi u}{\partial \bar{z}}=g .
$$

We know that $\frac{\partial x u}{\partial \bar{z}} \in L^{2}$. So if $c_{0}<1,[\mathbf{1}+\alpha S]^{-1}$ is invertible on $L^{2}$ (D) and $\frac{\partial \chi u}{\partial \bar{z}}=[\mathbf{1}+\alpha S]^{-1} g$. But if moreover $(|\alpha| \leq) c_{0}<\frac{1}{\epsilon_{r}}$ then this $L^{2}$ inverse (given by $\left.\sum(-1)^{k-1}(\alpha S)^{k}\right)$ maps $L^{r}$ into $L^{r}$, so $\nabla(\chi u) \in L^{r}$. (Sikorav seems to give an argument with less care.)

Step 4. We now claim that $\Delta(\varphi \circ u) \in L_{\mathrm{loc}}^{r / 2}(\Omega)$, where $\varphi=\operatorname{Re} z_{j}$ or $\operatorname{Im} z_{j}$ as in step 1 .

Indeed off $E$ we have

$$
|\Delta(\varphi \circ u)|=\left|d d_{J}^{c} \varphi\left(\frac{\partial u}{\partial x}, J(u) \frac{\partial u}{\partial x}\right)\right| \leq C\left|\frac{\partial u}{\partial x}\right|^{2} .
$$


As shown in step $1, \Delta(\varphi \circ u)$ is a measure on $\Omega$, we now only need to check that it has no mass on $E$. It is given by Lemma 2.3.

The end. Fix $\infty>r>4$. Since $\Delta u \in L_{\mathrm{loc}}^{r / 2}(\Omega)$ then $\nabla u \in C^{1-\frac{4}{r}}(\Omega)$. So $u \in C^{1}$ on $\Omega$ and therefore by continuity $u$ is $J$-holomorphic on $\Omega$.

\section{Proof of Theorem B implying Theorem C}

Recall that we are given the following: $\mathscr{C}$ is a closed subset of an almost complex manifold $(M, J)$. Assume that there is a discrete subset $S$ of $\mathscr{C}$ such that $\mathscr{C} \backslash S$ is locally exact $J$-pluripolar. $\Omega$ is a connected open subset of $C$ and $u: \Omega \rightarrow M$ is a continuous map. Assume that $u$ is $J$-holomorphic on $\Omega \backslash u^{-1}(\mathscr{C})$. Assume that there exists $z_{0}$ with $u\left(z_{0}\right) \notin \mathscr{C}$. We want to show that $u$ is $J$-holomorphic.

Let $V$ be the set of $z \in \Omega$ such that

a) $u$ is $J$-holomorphic on a neighborhood of $z$

b) $u(z) \notin S$ (the exceptional discrete set)

c) no neighborhood of $z$ is mapped into $\mathscr{C}$.

Let $V_{0}$ be the connected component of $V$ containing $z_{0}$. We claim that $V_{0}$ is an open and relatively closed subset of the open set

$$
X=\{z \in \Omega ; u(z) \notin S\} .
$$

Openness requires a justification because of $\mathrm{c}$ ). If $z_{1} \in V$ there exists a neighborhood $W$ of $u\left(z_{1}\right)$ and a $J$-plurisubharmonic function $\rho$ in $W$, continuous as a map into $\{-\infty\} \cup \mathrm{R}$, such that $\rho^{-1}(-\infty)=\mathscr{C} \cap W$ (possibly empty). Then $\rho \circ u$ is subharmonic near $z_{1}$ and not identical to $-\infty$. Hence $(\rho \circ u)^{-1}(-\infty)$ has empty interior. No open subset of some neighborhood of $z_{1}$ can be mapped into $\mathscr{C}$.

Now we check that $V_{0}$ is a relatively closed subset by the same argument. If $z_{1} \in X \cap \bar{V}_{0}$, take $\rho$ as before $\rho \circ u$ is subharmonic (because it is subharmonic when it is not $-\infty)$, so the set of $z$ such that $u(z) \in \mathscr{C}$ is a closed polar set in a neighborhood of $z_{1}$. By Theorem B, $u$ is $J$-holomorphic at $z_{1}$. So a) is proved and c) is trivial.

Therefore, $V_{0}$ is a connected component of $X$. If $z_{2} \in \Omega$ is on the boundary $b V_{0}$ one must have $u\left(z_{2}\right) \in S$, by the definition of $X$.

Fix $z_{2} \in \Omega \cap b V_{0}$. Since $S$ is discrete, there exists $\epsilon>0$ such that $u(z) \equiv p \epsilon$ $S$ for all $z \in b V_{0}$ such that $\left|z-z_{2}\right|<\epsilon$. Let $L$ be a $J$-plurisubharmonic function defined near $u\left(z_{2}\right)$ with pole $(-\infty)$ at $u\left(z_{2}\right)$ (Chirka's function, continuous 
away from $\left.u\left(z_{2}\right)\right)$. Shrinking $\epsilon$ if needed we can assume that $r(z)=L \circ u(z)$ is defined on the disc $\left\{z \in \mathrm{C} ;\left|z-z_{2}\right|<\epsilon\right\}$, where on that disc

$$
r(z)=L \circ u(z) \text { if } z \in V_{0}, \quad r(z)=-\infty \text { if } z \notin V_{0} .
$$

$r$ is a subharmonic function and $r^{-1}(-\infty)$ is a closed polar subset, which is removable for $u$ by Theorem $\mathrm{B}$.

Therefore, $u$ is $J$-holomorphic at each point of $\Omega \cap b V_{0}$ (and $J$-holomorphic on $V_{0}$ by definition). And $\Omega \backslash V_{0}$ has empty interior. Otherwise, by the connectedness of $\Omega$ the interior of $\Omega \backslash V_{0}$ has a boundary point $z_{2} \in b V_{0} \cap \Omega$, and the above polar set $r^{-1}(-\infty)$ has non-empty interior, which is a contradiction. This shows that $u$ is $J$-holomorphic on $\Omega$.

\section{REFERENCES}

1. Ahlfors, L. V., Lectures on Quasiconformal Mappings, Van Nostrand Mathematical Studies, No. 10 D. Van Nostrand Co., Inc., Toronto, Ont.-New York-London 1966.

2. J.-P. Demailly, Estimations $L^{2}$ pour l'opérateur $\bar{\partial}$ d'un fibré vectoriel holomorphe semi-positif au-dessus d'une variété Kählérienne complète, Ann. Sci. École Norm. Sup. (4) 15 (1982), no. 3, 457-511.

3. Diederich, K., and Fornæss, J. E., Pseudoconvex domains with real-analytic boundary, Ann. of Math. (2) 107 (1978), no. 2, 371-384.

4. Floer, A., Hofer, H., and Salamon, D., Transversality in elliptic Morse theory for the symplectic action, Duke Math. J. 80 (1995), no. 1, 251-292.

5. Henkin, G., and Leiterer, J., Theory of Functions on Complex Manifolds, Monogr. Math. 79 (1984).

6. Ivashkovich, S., and Rosay, J.-P., Schwarz-type lemmas for solutions of $\bar{\partial}$-inequalities and complete hyperbolicity of almost complex manifolds, Ann. Inst. Fourier (Grenoble) 54 (2004), no. 7, 2387-2435.

7. Ivashkovich, S., and Shevchishin, V., Structure of the moduli space in a neighborhood of a cusp-curve and meromorphic hulls, Invent. Math. 136 (1999), no. 3, 571-602.

8. Narasimhan, R., Introduction to the Theory of Analytic Spaces, Lecture Notes in Math. 25 (1966).

9. Pali, N., Faisceaux $\bar{\partial}$-cohérents sur les variétés complexes, Math. Ann. 336 (2006), 571-615.

10. Rosay, J.-P., J-holomorphic submanifolds are pluripolar, Math. Z. 253 (2006), no. 4, 659665.

11. Sikorav, J.-C., Some properties of holomorphic curves in almost complex manifolds in Holomorphic curves in symplectic geometry, Progr. Math. 117 (1994), 165-189.

DEPARTMENT OF MATHEMATICS

UNIVERSITY OF WISCONSIN

MADISON, WI 53706

USA

E-mail: gong@math.wisc.edu,jrosay@math.wisc.edu 